\title{
SARS-CoV-2: Emerging Role in the Pathogenesis of Various Thyroid Diseases
}

\author{
Avaniyapuram Kannan Murugan (D) \\ Ali S Alzahrani ${ }^{1,2}$ \\ 'Division of Molecular Endocrinology, \\ Department of Molecular Oncology, King \\ Faisal Specialist Hospital and Research \\ Centre, Riyadh, I I I I, Saudi Arabia; \\ ${ }^{2}$ Department of Medicine, King Faisal \\ Specialist Hospital and Research Centre, \\ Riyadh, I I2II, Saudi Arabia
}

Correspondence: Avaniyapuram Kannan Murugan

Division of Molecular Endocrinology,

Department of Molecular Oncology, King

Faisal Specialist Hospital \& Research

Centre, Riyadh, II2II, Saudi Arabia

Tel +966 I I-4647272 Extn 35076

Email akmurugan@gmail.com

\begin{abstract}
Coronavirus disease-2019 (COVID-19) is asymptomatic in most cases, but it is impartible and fatal in fragile and elderly people. Heretofore, more than four million people succumbed to COVID-19, while it spreads to every part of the globe. Severe acute respiratory syndrome-coronavirus-2 (SARS-CoV-2) induces various dysfunctions in many vital organs including the thyroid by utilizing ACE2 as a receptor for cellular entry. Emerging reports clearly show the involvement of SARS-CoV-2 in diverse thyroid disorders. Thus, this review article aims to review comprehensively all the recent developments in SARS-CoV-2induced pathogenesis of thyroid diseases. The review briefly summarizes the recent key findings on the mechanism of SARS-CoV-2 infection, the role of ACE2 receptor in viral entry, SARS-CoV-2-activated molecular signaling in host cells, ACE2 expression in the thyroid, cytokine storm, and its vital role in thyroid dysfunction and long-COVID in relation to thyroid and autoimmunity. Further, it extensively discusses rapidly evolving knowledge on the potential part of SARS-CoV-2 in emerging various thyroid dysfunctions during and postCOVID-19 conditions which include subacute thyroiditis, Graves' diseases, Hashimoto's thyroiditis, thyrotoxicosis, and other recent advances in further discerning the implications of this virus within thyroid dysfunction. Unraveling the pathophysiology of SARS-CoV-2triggered thyroid dysfunctions may aid pertinent therapeutic options and management of these patients in both during and post-COVID-19 scenarios.

Keywords: thyroid, COVID-19, hyperthyroidism, hypothyroidism, SARS-CoV-2, ACE2, inflammation, IL6, subacute thyroiditis, Graves' disease, Hashimoto's thyroiditis, thyrotoxicosis, p38/MAPK, thyroid cancer
\end{abstract}

\section{Introduction}

A novel sense-stranded RNA-containing virus was initially identified from China at the end of 2019 (31st December) called "severe acute respiratory syndrome-coronavirus-2" (SARS-CoV-2). It is the root cause for abrupt but severe pulmonary illness in humans that has emerged as a pandemic health issue, named "coronavirus disease-2019" (COVID-19). ${ }^{1}$ From its primary outbreak, this could reach more than 200 nations globally. As of 1st October 2021, more than 4,786,203 lives succumbed to COVID-19 disease while it has infected 233,908,734 people worldwide. ${ }^{2}$ Although primarily a respiratory infection, SARS-CoV-2 affects many organs such as the brain, lungs, heart, kidney, colon, adrenal glands, thyroid, etc. This multi-organ involvement often leads to death. ${ }^{3,4}$ The paucity of research findings in the literature on the SARS-CoV-2 infection and its downstream effects remains a major limitation in establishing treatment options for this deadly infection. This review briefly describes the mechanism of human cellular infection of SARS-CoV- 


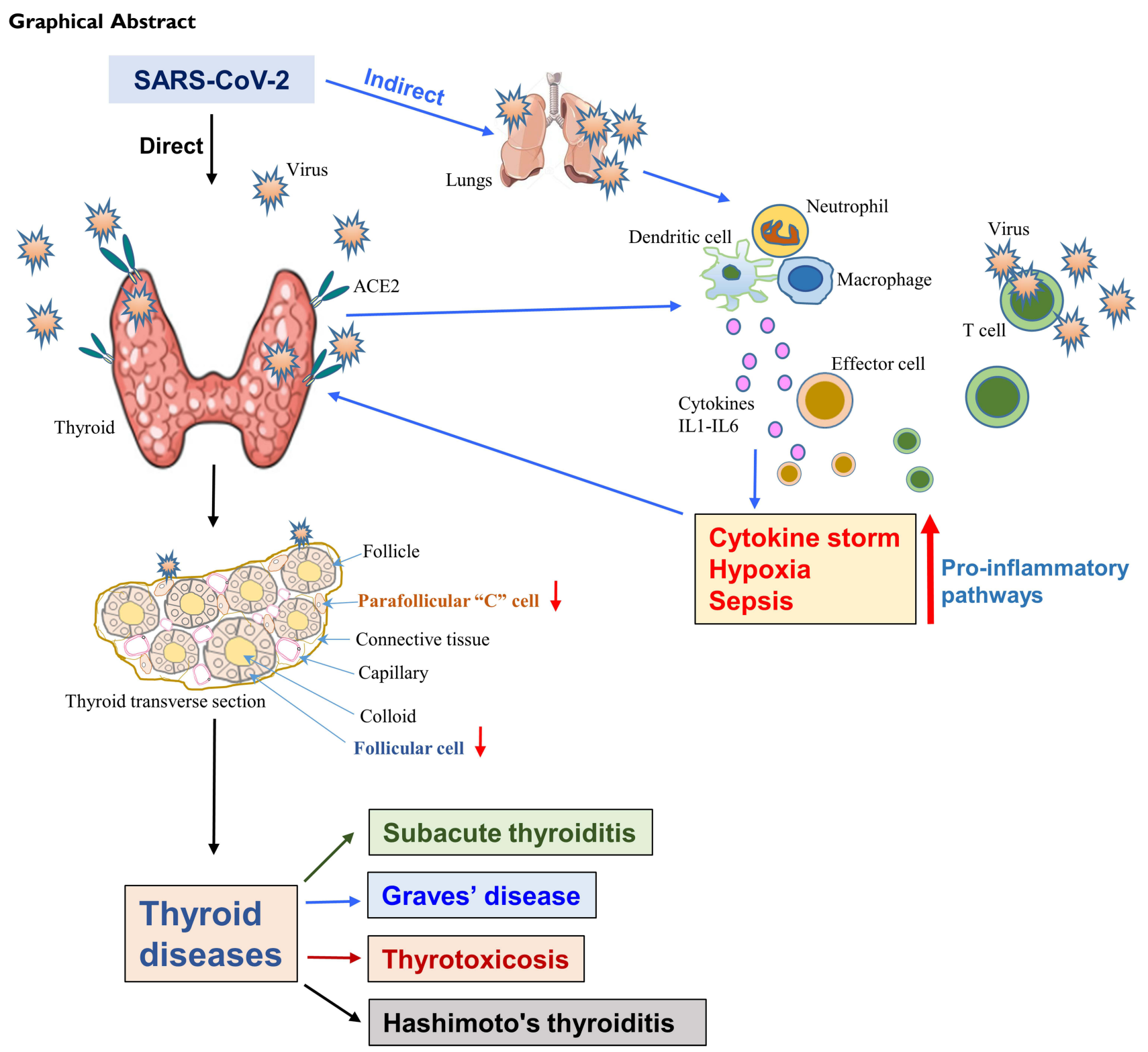

2 and discusses in detail virus-induced pathogenesis causing various thyroid diseases.

\section{Molecular Mechanism of Cellular Infection of SARS-CoV-2: The Mediator, ACE2 and Its Role}

Spike glycoprotein (S), 180-kDa protein (1273 amino acids) that generally occurs as trimeric form and it is a part of envelope protein and projected externally to form a structure similar to "corona" that facilitates the SARS-
CoV-2 entry. The virus hijacks human angiotensin-converting enzyme 2 (ACE2) to play the receptor-like role for its initial cellular entry, followed by efficient binding and membrane fusion (Figure 1). Particularly, the ectodomain of the "S" protein was shown to tightly bind to the ACE2 at the peptidase domain (PD). ${ }^{5}$ Similarly, the ACE2 was also demonstrated as a viral entry-receptor for another variant, SARS-CoV-1 in early $2003 .{ }^{6}$

The ACE2 is a transmembrane type I glycoprotein (mono-carboxypeptidase) localized on the cell surface and contains 805 amino acids which are primarily involved in 
A

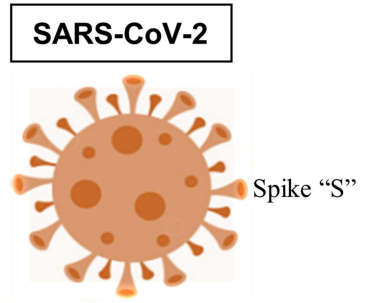

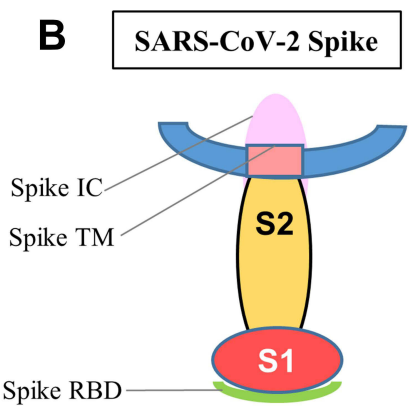

C SARS-CoV-2 Spike- Ectodomain

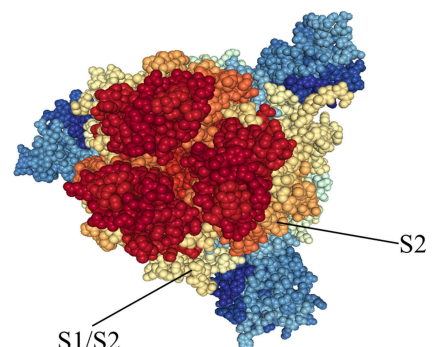

D

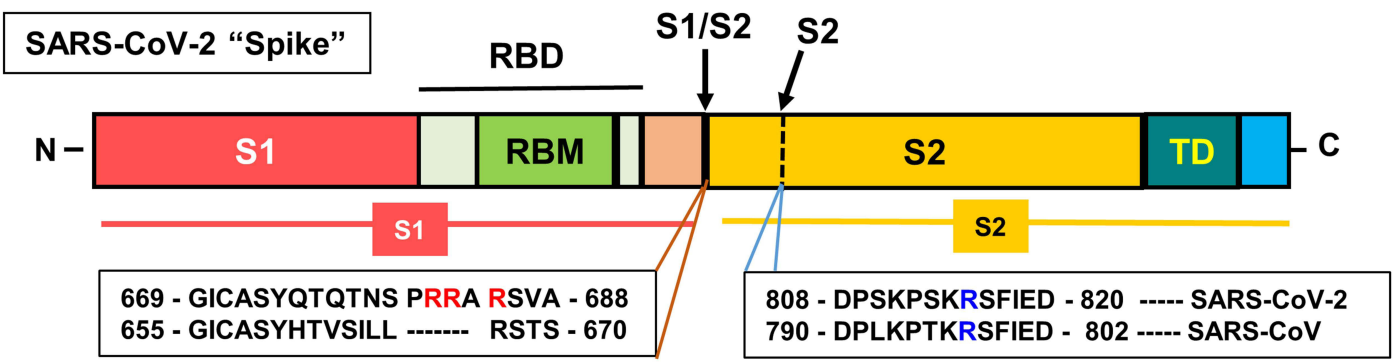

E

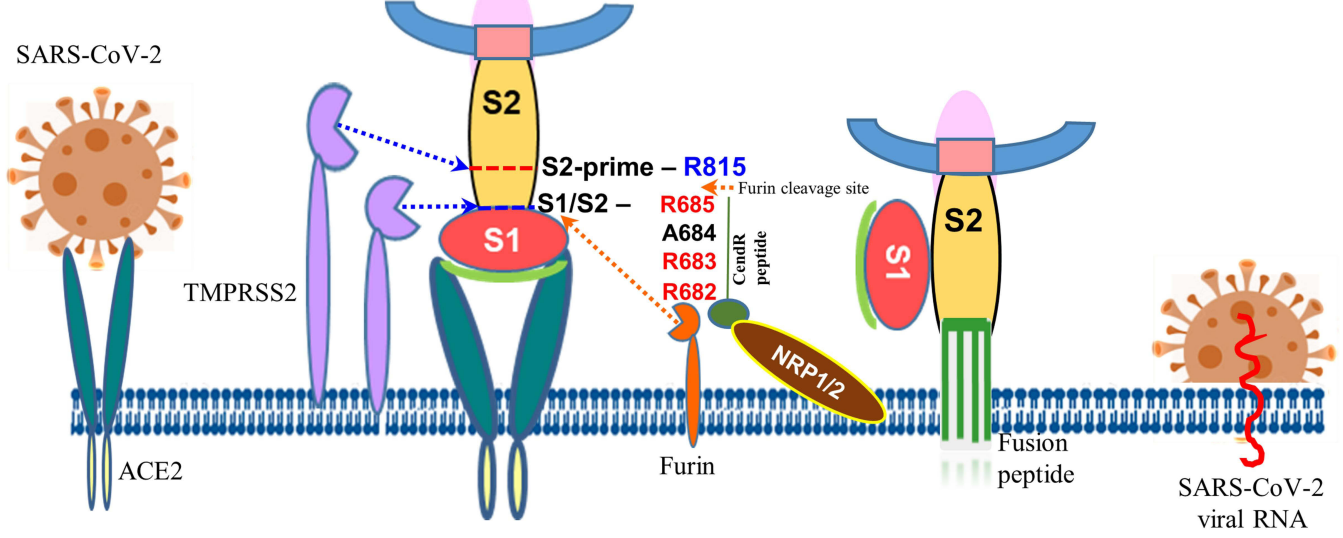

$\mathbf{F}$

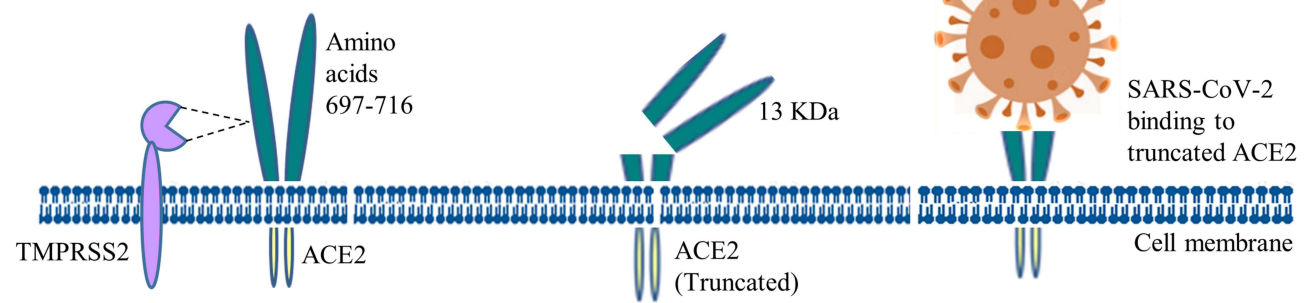

Figure I Molecular mechanism of SARS-CoV-2 infection. (A) Schematic diagram of SARS-CoV-2. (B) "Spike" protein structure and its various operative parts. (C) Cryo-EM structure of "spike" - ectodomain was visualized, SI/S2 junction and S2 cleavage sites were marked by NGL viewer (PDB ID: 7CN9). (D) Schematic diagram of "spike" protein (S) with various functional domains. (E) The diagram shows the entry of SARS-Co-V-2 into a host cell. "Spike" recognizes ACE2 protein and binds to it via the SI subunit. Subsequently, the SI is cleaved at the SI/S2 junction with unique amino acid motifs, R682, R683, and R685, or within the S2 subunit by TMPRSS2 and furin that facilitates the structural restriction of SI on S2. Neuropilin-I (NRPI) was shown to attach furin-cleaved substrates (SI) in the " $\mathrm{C}$-end rule" CendR motif that potentiates SARS-CoV-2 infectivity. This process releases the internal fusion peptide attached to the spike TM domain for successful viral and cellular membrane fusion. This enables the viral genome to enter the host cell. (F) Diagrammatic representation of TMPRSS2mediated cleavage of ACE2. The TMPRSS2 could cleave ACE2 at amino acids 697-716 and that results in chopping of a I3 kDa ACE2 fragment and facilitate viral infectivity. The fragment can be easily detected in the cell culture medium. 
angiotensin (Ang) ontogenesis. The peptide-based Ang hormone directly controls vasoconstriction and enhances pressure in the blood vessels. The ACE2 was initially discovered by two groups independently in the year 2000., ${ }^{7,8}$ ACE2 protein has two major domains: of which, a peptidase domain (PD) is located at the N-terminal while a collectrin-like domain (CLD) is found at C-terminal which is ending with a transmembrane helix that includes an intracellular portion approximately with forty residues. ${ }^{9}$ The PD of ACE2 plays a primary role in cleaving Ang I to yield Ang-(1-9), resulting biomolecule is secondly processed by other enzymes (ACE, angiotensin-converting enzyme, and neprilysin, zinc-dependent metalloprotease) to become Ang-(1-7), a vasodilatory heptapeptide. The ACE2 can also directly cleave the robust molecule of vasoconstriction, Ang II to give Ang-(1-7). ${ }^{8,9}$ Although ACE2 catalyzes both Ang II and Ang I, it much more efficiently catalyzes only Ang II which is 400 times higher than Ang I. ${ }^{10}$ The ACE2 also has potent cleaving activity on other peptides which include apelin-13, apelin36, $\beta$-casomorphin, des-Arg9-bradykinin, dynorphin, and neocasomorphin. ${ }^{11}$ ACE-inhibitors are widely used as antihypertensive drugs but they usually do not have the ability to inhibit ACE2 due to distinct structural variation in the catalytic domain even though ACE2 exhibits $~ 40 \%$ structural similarity with ACE. ${ }^{7}$

Two important steps are comprised in SARS-CoV-2 infection which consists of initial recognition of the receptor (ACE2) and efficient fusion of cell membrane. The viral "Sprotein" on the exterior portion of SARS-CoV-2 initiates the receptor identification and fusion of the cell membrane process. The viral "S-protein" complex consists of heterodimeric proteins namely "S1" and "S2" (subunits 1 and 2, respectively). The "S1" comprises a receptor-binding domain (RBD) which could directly bind with the ACE2 via peptidase domain (PD). Subsequently, receptor transmembrane protease serine 2 (TMPRSS2) chops "spike" at two points $\mathrm{S} 1 / \mathrm{S} 2$ (a point of $\mathrm{S} 1$ and $\mathrm{S} 2$ subunit intersection) and S2 (a restriction site within the S2 subunit) and this step is critical for cellular viral entry. ${ }^{11}$ The TMPRSS2 gene is mostly regulated by androgen and highly expressed in the prostate while minimally expressed in the other organs including the colon, kidneys, liver, lung, and pancreas. It has been localized in extracellular vesicles even though the TMPRSS2 is mainly membrane-bound. ${ }^{12}$ This indicates that the protease could get into other tissues away from its originally expressed sites to facilitate the ACE2 proximity in viral infection. Moreover, TMPRSS2 is shown to be stoichiometrically contiguous to ACE2. ${ }^{13}$ Right after the S1 subunit detachment from the "S" protein, the remaining viral S2 subunit goes through a conformational change which exposes the transmembrane (TM) domain of S2 to the host cellular membrane. This results in viral transmembrane domain fusion with the host cellular membrane which triggers infusing the viral particle and release of its content including the RNA into the cell that drives subsequent replication and infection to other cells. The TMPRSS2 was demonstrated to be critical also for other coronaviruses and influenza viral infection while only the proximity of both the ACE2 and TMPRSS2C could facilitate the viral infection. Camostat mesylate, a TMPSRR2 inhibitor and was shown to block the priming of " $\mathrm{S}$ " protein and partly inhibit the subsequent entry of SARS-CoV-2. ${ }^{14}$ Furin, a cellular protease has been demonstrated to cleave the spike glycoprotein at the $\mathrm{S} 1 / \mathrm{S} 2$ junction resulting in two associated polypeptides, $\mathrm{S} 1$ and S2 which were shown to be critical for spike-protein (S)mediated cell-cell fusion and human cell entry. ${ }^{15}$ Neuropilin1/2 (NRP1/2), transmembrane receptors implicated in angiogenesis, axon guidance, and vascular permeability and are highly expressed in respiratory and olfactory epithelium while epithelial and endothelial cells show foremost. Recently, NRP1/2 has been demonstrated to bind directly furin-cleaved S1 fragment of the spike protein at the " $\mathrm{C}$ end rule" (CendR), a carboxyl (C)-terminus (R/K-XX-R/K) motif-containing key array of amino acids (682_R-R-AR_685) could potentiate SARS-CoV-2 infectivity. Smallmolecule inhibitor or monoclonal antibodies-mediated inhibition of this interaction was shown to suppress the viral infection. $^{16,17}$

Apart from the SARS-CoV-2, the other two members of the same family (coronaviridae) including SARS-CoV, and NL63 exploit the ACE2 as a receptor for their human cellular entry. ${ }^{18}$ Both SARS viruses (CoV-1 and CoV-2) show $\sim 80 \%$ amino acid conservation between them suggesting that their natural tendency in binding affinity towards ACE2. Conversely, among the human population, the presence of some natural single nucleotide polymorphisms (SNPs) (rs73635825 (S19P) and rs143936283 (E329G) in ACE2 was shown to reduce the binding affinity towards the viral " $\mathrm{S}$ " protein suggesting natural protection against pandemic COVID-19. ${ }^{19}$ The ACE2 has a major role in the "compensatory axis" of the RAAS (ACE2-Ang (1-7)-Mas), a vasodilator signaling, counteracting the effects of the classical pathway for vasoconstriction (ACE/Ang-II/AT1 receptor), in regulating blood pressure, chronic heart failure, and cardiac hypertrophy. ${ }^{20}$ 
Deregulated Pathways in COVID-I 9 Disease: The Potential Therapeutic Targets

The rapid development of antiviral therapies is a necessity for nations across the globe. Inadequacy of empirical scientific literature on the genetic and biochemical signature of SARS-CoV-2-mediated host cell signaling prevents the development of antiviral therapies. Recently, a study performed phosphoproteomic experimentation of SARSCoV-2-infected host cells (Vero E6) utilizing mass spectrometry and revealed various unique phosphorylated protein molecules invariably in both monkey cells (host) and viruses. Among them, viral infection mainly activated crucial cellular signaling networks include p38 MAPK, a class of MAPKs that could respond to various stimulus factors (cytokines, heat and osmotic shock, and UV) and casein kinase 2 (CK2) pathways. This p38 MAPK is mainly required for cellular differentiation, growth, development, apoptosis including autophagy. Further, viral protein promoted the development of various cytokines and blocked many mitotic kinases, which collectively could result in cell cycle arrest. The SARS-CoV-2 infection also induced the CK2-mediated filopodial formation that facilitated the process of budding viral particles as illustrated in Figure 2. The study also targeted the deregulated kinase pathways with many drugs that revealed therapeutic targeting of the p38, CK2, cyclin-dependent kinase (CDK), tyrosine-protein kinase receptor UFO (AXL), and 1-phosphatidylinositol 3-phosphate 5-kinase (PIKFYVE) kinases could display a potent antiviral efficacy against the COVID-disease. ${ }^{21}$ Similarly, another study also performed phosphoproteomics analysis to determine signaling changes in the virus-infected host cell. They discovered changes in phosphorylation of viral proteins that phospho-

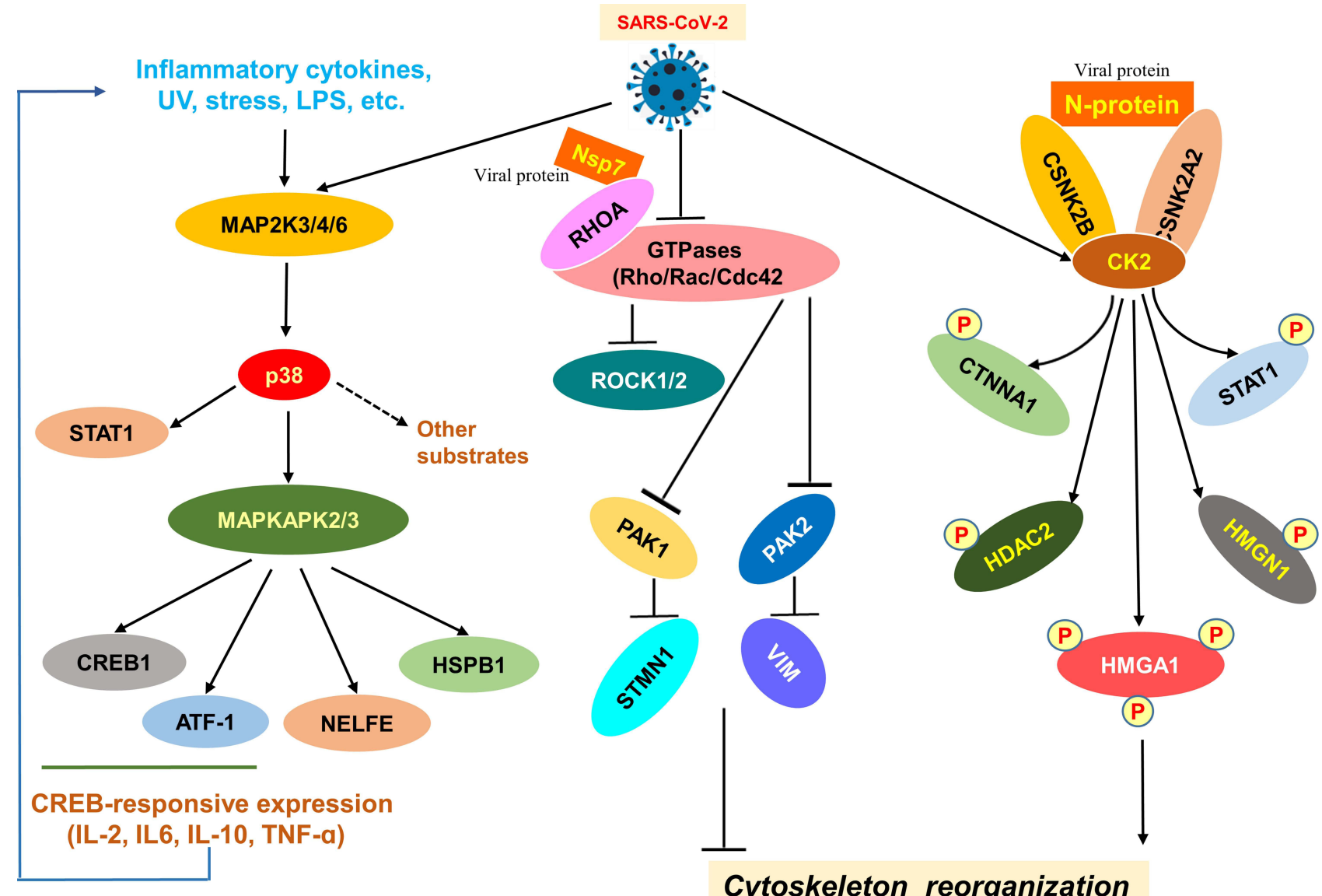

p38/MAPK pathway

Rho/Rock signaling

Casein Kinase II signaling

Figure 2 SARS-CoV-2-induced signaling pathways. Diagram of the SARS-CoV-2-mediated p38/MAPK, Rho/Rock, and casein kinase II signaling pathways involved mainly in the viral replication. The P38/MAPK pathway executes a vital role in cell proliferation while the Rho/Rock, and casein kinase II signaling pathways are implicated in the cytoskeleton reorganization of normal cells. The Rho/Rock signaling is reduced upon infection. 
driven deregulated cellular pathways in the host. The findings revealed that growth factor receptor (GFR) signaling particularly the PI3K and MAPK are hyperactivated (Figure 2). Further, various drug-protein network analyses identified the GFR pathway as the key target for antiviral therapeutic intervention. Inhibition of downstream of GFR signaling using five drug molecules inhibited mainly the viral dsRNA synthesis, and release of viral RNA into the supernatant ultimately resulting in suppression of the SARS-CoV-2 replication in cells suggesting that the GFR pathway is crucial for viral replication. ${ }^{22,23}$ These key studies demonstrate that GFR-related p38MAPK and PI3K signaling is important targets in seeking therapeutic intervention. Like other organs and organ systems, the GFR-related MAPK and PI3K signaling pathways and cytoskeleton modifying proteins implicate in potential aspects also in the normal cellular functions of the thyroid gland. Given the importance of these signaling, it is plausible that SARS-CoV-2-mediated deregulation of these pathways could cause thyroid dysfunction upon viral infection.

\section{ACE2 is Highly Expressed in the Thyroid Gland}

A recent study performed the expression analysis of ACE2 in thirty-one tissues derived from normal people and compared males versus females, younger versus older people within that tissue samples. Besides, the study also correlated the expression of ACE2 versus enrichment levels of "immune signature" within each tissue. The study found that ACE2 protein expression is widely detected in different kinds of tissues including lung tissue. ACE2 expression was observed to be upregulated within thyroid tissues in addition to the cardiac, renal, small intestine, testis, and adipose tissue. ${ }^{3,4} \mathrm{~A}$ medium level of ACE2 expression was detected in the adrenal gland, bladder, colon, liver, and lungs while the minimal level was seen in the brain, blood vessels, blood, bone marrow muscle, and spleen. ${ }^{3}$ In thyroid, enrichment analysis manifested that the expression level of ACE2 was inversely connected with the "killer" $\mathrm{T}$ cell $\left(\mathrm{CD}^{+}\right)$in the feminine gender. Expression levels of ACE2 and interferon response makeup in the thyroid were significantly associated favorably in masculine and inversely in feminine genders. Moreover, no difference in the expression levels of ACE2 was found regardless of gender and age difference in many other tissues. ${ }^{3}$ These results suggest that not only the lungs but also the thyroid is a bullseye for SARS-CoV-2 infection next to its related pathogenesis (Figure 3). Similarly, the TMPRSS2 also displays a top-level of its mRNA in the thyroid tissue without reference to gender difference. ${ }^{24}$ This once again may facilitate the viral infection to the thyroid that could lead to thyroid dysfunction during and post-COVID-19 cases.

\section{SARS-CoV-2 Infection and Cytokine Storm: A Vital Role in Thyroid Dysfunction}

A recent systematic review analyzed seven independent reports consisting of 1237 cases with COVID-19 which clearly revealed the presence of thyroid dysfunction in a significant percentage of COVID-19 cases. $^{25}$ In addition, thyroid dysfunction in $\sim 15 \%$ of mild-to-moderate cases of COVID-19 and incident thyroid dysfunction and autoimmunity in COVID-19 survivors have recently been observed. $^{26,27}$ As illustrated in Figure 3, the thyroid gland is likely dysfunctional as a consequence of either direct or indirect effects of SARS-CoV-2 infection. The thyroid gland can be directly infected by the SARS-CoV-2 as the ACE2, TMPRSS2, and other factors facilitating viral entry are highly expressed in it. $^{3}$ Consistently, the SARS-CoV-2 RNA has recently been detected within the thyroid gland and it supports the notion that direct infection of the thyroid gland by SARS-CoV-2. ${ }^{28}$ This infection could induce cytokine storm which in turn may attack the thyroid gland. On the other hand, the thyroid gland is indirectly affected when the lung/respiratory systemmediated SARS-CoV-2 infection triggers cytokine storm and this could affect the thyroid gland. Cytokine storm plays a key role in both direct and indirect effects on the thyroid gland. Cytokine storm - a condition wherein uncontrolled over-responsive immune, inflammatory, coagulation, and complement systems cause robust secretion of proinflammatory cytokines in the bloodstream much higher than normal level often due to pathogenic infection. $^{29}$ This cytokine storm triggers epithelial and endothelial cell injuries by inducing intensive apoptosis that leads to vascular leakage mainly due to enhanced permeability resulting in multiple organ failures. ${ }^{30}$ This hyperactivation and hypersecretion of immune and/or inflammatory cytokine molecules could cause a hyperinflammatory condition that induces not only severe damage to many vital organs but also causes death which has been frequently associated with the COVID-19. ${ }^{30-32}$ Although 


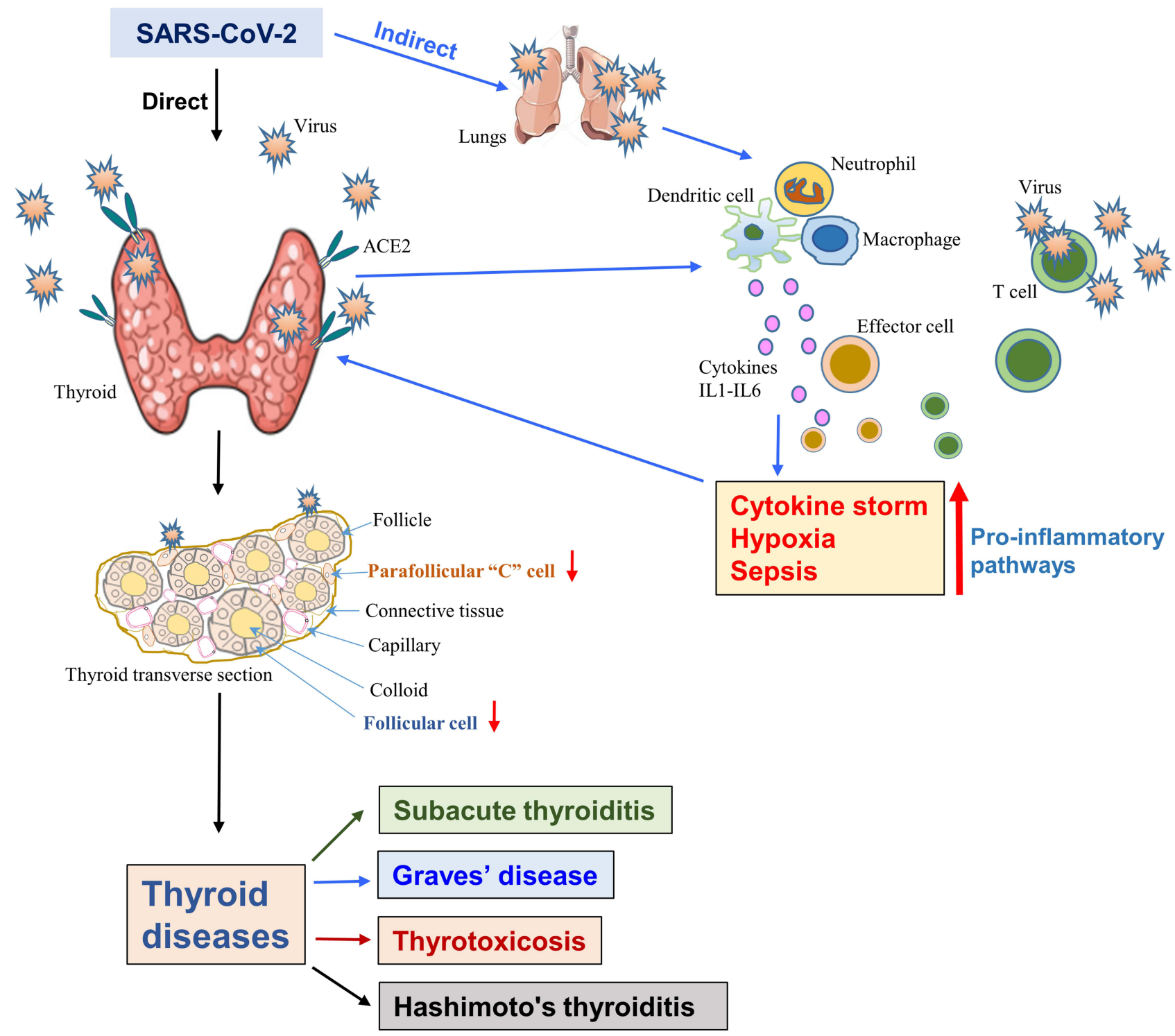

Figure 3 The SARS-CoV-2-induced thyroid dysfunction. The diagram displays both the direct infection of the thyroid as the viral receptor, the host protein ACE2 is highly expressed in the thyroid. Direct viral infection damages both the follicular and parafollicular cells that result in thyroid dysfunctions. Alternatively, host lung and other organmediated infections induce a "cytokine storm" due to the activation of various pro-inflammatory pathways, which in turn, affects the thyroid and causes its dysfunction.

many cytokines (secreted by dendritic cells and mononuclear macrophages) such as interleukins (IL1, IL6, IL8, and IL17)), chemokines (CCL2-CCL4, CCL8-CCL10), tumor necrosis factor-alpha (TNF- $\alpha)$ and interferon (IFN)- $\gamma$ have been shown to be involved in a cytokine storm, only some of them (IL1 $\beta$, IL6, TNF- $\alpha$, and IFN- $\gamma$ ) are mainly implicated in COVID-19-induced cytokine storm. ${ }^{30,33-35}$

The molecules that activate and boost innate immune signaling are called damage-associated molecular patterns (DAMPs). They are classified into five classes based on their receptor/sensors and are released from the injured cells/tissues include mtDNA and cytosolic RNA, heat shock proteins (HSPs), eATPs, ROS, actin cytoskeleton, oxidized proteins/lipids, etc. ${ }^{30}$ The DAMPs function as ligands and are sensed by various mononuclear macrophage and dendritic cell surface receptors (pattern recognition receptors, PRRs: toll-like receptor, TLR, and retinoic acid-inducible gene-1-like receptor, RLR). Upon activation, these receptors induce and orchestrate inflammation response against infection by activating key transcription factors of immune-related signaling pathways which cause upregulation of cytokines, chemokines, and adhesion molecule-coding genes. ${ }^{30,36}$ This hyperactivated proinflammatory cascade of cytokines triggers acute-phase responses via inducing innate immunity and the cytokines 
also stimulate infiltration and transportation of monocytes and $\mathrm{T}$ lymphocytes to the infected site. ${ }^{36,37}$ The IL6 and IFN- $\gamma$ are mainly involved in adaptive immunity ( $\mathrm{T}$ cellmediated) wherein, particularly $\mathrm{T}$ helper lymphocytes (Th1, Th2 and Th17) and CD8+ T cells, cytotoxic $\mathrm{T}$ lymphocytes (CTLs) have been shown to be implicated in immune defense towards intracellular pathogens (viruses and bacteria). Increased immune responses of $\mathrm{T}$ helper lymphocytes (Th1/Th17) and IL17-mediated cytokine signaling have been detected in SARS-CoV-2 infections. $^{32,38-40}$ Interestingly, these immune factors were shown to be associated with various autoimmune endocrine diseases, including autoimmune thyroid disorders. ${ }^{41,42}$

As observed in COVID-19, cytokine activation is also implicated in various autoimmune-mediated thyroid disorders suggesting viral infection may play a role in latent or new-onset autoimmunity. ${ }^{41,43}$ The viral infection-mediated hyper-inflammatory condition is likely to set off an immunological avalanche that could activate the autoimmune hyperthyroidism and the enhanced levels of IL-6 detected in Graves' disease reflect this phenomenon. ${ }^{43}$ Consistently, the COVID-19-mediated hyper-inflammatory condition was reported to be modulated mainly by Th1 and IL- $6 .{ }^{44}$ Nevertheless, the pathogenesis of autoimmune hyperthyroidism was found to be regulated by the autoimmune response of Th2. ${ }^{45}$ Moreover, Th1 and Th2 imbalance and enhanced production of Th1 and Th17 in peripheral lymphocytes and enhanced level of Th1/Th17-associated cytokines (IL17, IL21-IL23, IFN- $\gamma$, and TNF- $\alpha$ ) in serum have been documented in autoimmune thyroid disease cases. ${ }^{46-53}$ The activity of Th17 or Th1 has been postulated to be responsible for the development and progression of autoimmune disease. ${ }^{54}$

However, the current knowledge on Th17 and Th22 and their cytokines in the pathogenesis of autoimmune thyroid disease recommend revisiting the Th1 and Th2 role in the pathophysiology of thyroid disorders including Graves' hyperthyroidism and Hashimoto thyroiditis. ${ }^{53}$ Recently, the possible pathogenic mechanisms of immune-mediated thyroid disorders associated with SARS-CoV-2 infections are extensively discussed by Ruggeri et al. ${ }^{42}$ A clinical point of view on COVID-19related thyroid disorders was precisely documented by Giovanella et al. ${ }^{55}$ They suggest for future longitudinal studies to rule out the long-term viral effect on the thyroid gland. Further, it would also be interesting if future studies recapitulate these clinical findings in experimental settings, in vitro and in vivo that may pave the way for new avenues towards molecular therapies targeting these disorders. Moreover, data on SARS-CoV-2/COVID-19induced thyroid dysfunctions or thyroid pathology are emerging and they are discussed in the subsequent topics.

\section{Long-COVID: Thyroid Gland and Autoimmunity}

The majority of the COVID-19 cases completely recover in less than 12 weeks after initial infection but some of them have persistence of a broad range of symptoms that last many weeks or months even after recovery and this event is called post-acute sequelae of SARS-CoV-2, postCOVID-19 syndrome, post-acute COVID-19 syndrome, and most commonly it is termed as "long-COVID". ${ }^{56}$ As cases of COVID-19 recovery is increasing, there is a chance to evolve the long-COVID as a pandemic of the "pandemic". ${ }^{66}$ Long-COVID cases may show various symptoms including fatigue $(53 \%)$, shortness of breath (43\%), joint pain $(27 \%)$, chest pain $(22 \%)$, low vision $(17 \%)$, cough (16\%), loss of smell (15\%), Sjögren's syndrome (14\%), rhinitis (13\%), loss of taste $(10 \%)$, red eyes $(10 \%)$, lack of appetite $(8 \%)$, headache $(9 \%)$, sputum production $(8 \%)$, lack of appetite $(8 \%)$, skin lesions $(7 \%)$, sore throat $(7 \%)$, vertigo $(6 \%)$, muscle pain $(6 \%)$, and diarrhea (3\%) and other unidentified issues. ${ }^{57}$ The logCOVID may develop due to either persistence of residual issues even after recovering from acute infection or new issues/syndromes may develop even though initially had mild/asymptomatic COVID-19. ${ }^{58,59}$

The COVID-19 affects many organs and organ systems thus it is plausible that the sequelae can develop in many organs including the thyroid following COVID-19. ${ }^{56,60}$ Thyroid gland has gained considerable attention in relation to COVID-19 at both acute and convalescent-phase. The HPT axis has been clinically linked with the acute phase of COVID-19, in which a remarkable number of studies are accumulated particularly on nonthyroidal illness and thyroiditis. Nonetheless, only a few studies are documented on the status of the thyroid gland in the convalescent phase of COVID-19.

Initially, incident thyroid dysfunction and autoimmunity have been observed among 122 COVID cases during the convalescence phase. ${ }^{27}$ Subsequently, the same group evaluated both the prevalence and predicting factors of long-COVID and possible functions of thyroid and autoimmunity in long-COVID. The study included 250 adults 
with no previous history of thyroid disorders and performed thyroid function tests and antithyroid antibody measurements in all cases prior to admission and at follow-up (median: 89 days). Of these cases, $20 \%$ displayed long-COVID and observed positivity of incident antithyroid peroxidase (anti-TPO) though incident thyroid dysfunction was uncommon. These studies suggest that a possible role of thyroid autoimmunity after COVID-19 and also raise the possibility that anti-TPO could go ahead of thyroid dysfunction. However, further studies with more sample size and lengthy follow-up are required to give a fine conclusion. ${ }^{61}$

Furthermore, a recent case-control study found a significant correlation between hypothyroidism and prolonged SARS-CoV-2-induced anosmia and suggested that the smell dysfunction is likely due to the direct virusinduced damage on both the thyroid and olfactory nerve. ${ }^{62}$ Moreover, this no olfactory function or slow recovery is likely because of viral-induced thyroid damage resulting in depletion of thyroid hormones may impair the regeneration and maturation of olfactory neural cells at least in a subset of cases with thyroid dysfunction. These primary observations warrant prospective studies in the future to precisely evaluate the incidence and predictors of longCOVID and the role of thyroid function and autoimmunity.

\section{Thyroid Dysfunctions in COVID-I9 Cases}

The COVID-19 is a severe infectious and widespread disease that boasts massive consequences at various ranges on several organs including the thyroid. The SARS-CoV-1 also exploits similar infection machinery and causes more severe respiratory disease but it exhibits low infectivity. Previously, the SARS-CoV-1 has been shown to affect many organs including the thyroid. ${ }^{63,64}$ Triiodothyronine (T3) and thyroxine (T4) measurements were reported to be lower than controls in SARS-CoV-1-infected patients irrespective of phases (acute and convalescent) suggesting that the infected people have an underlying sick-euthyroid syndrome. Moreover, SARS patients in intensive care units with sick-euthyroid syndrome exhibited low thyroid weight due to reduced follicular size of thyroid which was also associated with colloid depletion. ${ }^{63}$ Further, an autopsy study conducted on five SARS patients displayed remarkable destruction of thyroid cells including follicular and parafollicular cells. ${ }^{64}$ The former type of cell damage is likely to cause a low T3 and T4 while the latter cell type (parafollicular) destruction could result in decreased calcitonin in serum. The SARS was shown to cause reversible central hypothyroidism at least in $5 \%$ of cases affecting/ deregulating the hypothalamic-pituitary-thyroid (HPT) axis function. ${ }^{65}$ This was mainly due to the chronic cortisol hypersecretion (secondary to viral infection) which may lead to a decreased hypothalamic-pituitary-adrenal (HPA) axis activity although transient. ${ }^{66}$ The HPA axis influences the HPT axis mainly in two ways which cause a differential effect. Firstly, acute stress-induced secretion of glucocorticoids directly stimulates pituitary thyrotrope that results in increases in circulating thyroid-stimulating hormone (TSH), which mediates transient activation of the HPT axis. Secondly, chronic/prolonged stress-induced secretion of glucocorticoids inhibits the production of thyrotropin-releasing hormone (TRH) in the hypothalamus and hence TRH fails to stimulate the release of TSH from the anterior pituitary that results in perpetually reduced HPT activity. Alternatively, enhanced intrahypothalamic corticotropin-releasing hormone $(\mathrm{CRH})$ secretion due to stress could enhance somatostatin secretion this may also reduce TSH production, despite its effect is weaker than growth factors. Besides, glucocorticoid-mediated stimulation of the HPA axis for a prolonged time also decreases the T4-to-T3 (biologically active) change. ${ }^{67}$

An earlier study showed that the follicular epithelial and parafollicular cells of the thyroid of SARS patients were significantly injured with apoptosis. ${ }^{64}$ Further, it has been shown that TSH, total T3 (TT3), and total T4 (TT4) in SARS patients display remarkably low compared to the control group in both the progression and recovery phases of SARS disease. ${ }^{68}$ Besides, the pattern of quantity and intensity of staining of TSH-positive cells were demonstrated to be reduced in the pituitary gland of SARS patients that reflects the depleted TSH level in SARS patients could be due to the changes in the pattern of TSH-secreting cells of the pituitary gland. ${ }^{69}$ It seems that not only the thyroid but also the pituitary fall as a direct victim of SARS by causing non-thyroidal illness syndrome (NTIS) or low T3 syndrome. This phenomenon is because of either a direct effect of the SARS virus on the cells of the pituitary or an indirect effect that could result from the hyperactivation of many circulating pro-inflammatory cytokines due to the SARS infection. ${ }^{70,71}$ The NTIS is an adaptive physiological mechanism instead of true thyroid dysfunction which is mainly characterized by a reduced level of T3 in serum with a decreased or inappropriately 
normal TSH levels. The T4 is likely to decrease with prolonged NTIS and/or increased severity. The NTIS or low T3 syndrome has been shown to be significantly correlated with poor prognosis and death risk. ${ }^{71}$ This phenomenon is mostly executed by the differential effect of inflammation-associated cytokines (IL1 $\beta$, IL6, IFN- $\gamma$, and $\mathrm{TNF} \alpha$ ) on the HPT axis via reducing the T4-to-T3 conversion and enhancing the T4 and T3 deactivation. ${ }^{73}$ This mechanism is exclusively carried out by decreasing the enzymatic activity of two important biochemical factors (5'-monodeiodinases, D1 and D2) actively involved in T4to T3 conversion and concomitantly fostering the biochemical activity of D3 deiodinase which is important for deactivating the T4 and T3. ${ }^{71}$ Besides, the accumulation of cellular oxidative stress because of enhanced production of reactive oxygen species (ROS) has been shown to be correlated to NTIS. ${ }^{74}$ On the other hand, the NTIS condition is more often considered a euthyroid sick syndrome - an adaptive response via compensatory axis that helps to reduce the metabolic rate and ATP utilization in severely sick conditions, regardless of acute and chronic phase. Moreover, it is plausible that SARS therapeutic could lead to broken/deregulated cellular signaling resulting in vital hormonal alteration beyond the normal physiological range in the pituitary-thyroid axis feedback loops. These results raise the feasibility that SARS-CoV2 infection tends to have a detrimental effect on the thyroid and its normal physiological function.

A recent study reported analyses of the clinical data of 50 COVID-19 patients encompassing the presence and absence of a previous thyroid disorder. This group was compared with a control group, healthy cases who came over the clinic to regular checkups and pneumonia patients without "COVID-19", but the extremity of the illness was comparable to that of COVID-19. The levels of thyroid hormone were measured in both during and post-COVID19 scenarios. This study found that the TSH levels of COVID-19 cases $(28 / 50,56 \%)$ were very lower than the values of the normal range. A significantly reduced thyroid hormone levels (TSH and TT3) have been detected in COVID-19 cases when compared with the control and pneumonia with no COVID-19 cases. Moreover, the study found that the TSH and TT3 levels were directly proportional to the disease severity. However, the TT4 level in COVID-19 patients did not exhibit any significant differences when compared to the control group. Despite no thyroid hormone replacement therapy, all the patients displayed normal values of thyroid hormones (TSH, FT3,
FT4, TT3, and TT4) after recovery and numerical values were similar to the control groups. Besides, the coexistence of thyroiditis leading to FT4 release from the thyroid gland, juxtaposed on an underlying non-thyroidal illness syndrome (NTIS), a condition known as T4thyrotoxicosis. ${ }^{75}$ Interestingly, atypical thyroiditis associated with the SARS-CoV-2 infection has recently been reported. Muller et al were the first to describe the presence of "atypical thyroiditis" developing during COVID19 , which is mainly characterized by "T4 thyrotoxicosis" due to the presence of thyroiditis juxtaposed to low T3 syndrome secondary to the severity of illness; this explains the presence of low TSH and FT3 levels associated with normal or elevated levels of FT4. This condition was named "atypical thyroiditis" since, differently from the classic form of subacute thyroiditis was characterized by the absence of neck pain, predominance among males, and correlated with the illness severity. ${ }^{76}$ The thyroid hormone changes (TSH and TT3) discovered in several cases of COVID-19 disease could be vital disease manifestations for the course of the COVID-19. The low level of TSH in the COVID-19 patients may also be exerted by another factor, chronic stress that could result due to glucocorticoids and hypoxemia as the majority of COVID-19 patients $(31 / 50)$ were treated with methylprednisolone $(57.3 \mathrm{mg} /$ day) although a low dose. As SARS-CoV-2 is highly conserved in basic structural elements and inducing pathogenesis with SARS-CoV, it is prone that SARS-CoV2 could attack TSH-secreting pituitary cells as it was previously reported in SARS patients in spite of the molecular mechanism remains largely unknown. On the other hand, some of the shortcomings such as failure to assess the FT3, FT4, and TSH at the time of admission, testing of thyroid hormones during the course of glucocorticoid medication, and lack of thyroid hormone profile for mild COVID-19 cases together do not qualify to exclude the impact of hormonal alterations in pituitary-thyroid axis loop. A recent study profiled the thyroid hormone levels in 100 COVID-19 patients for determining if they could predict all-cause mortality. Obtained results revealed that the level of FT3 was remarkably lower $(<3.10 \mathrm{pmol} / \mathrm{L})$ in severely ill COVID-19 cases compared to non-severely ill cases and it remarkably correlated with all-cause mortality. However, other thyroid functional characteristics including FT4, TSH, and FT3/FT4 were not significantly linked. ${ }^{77}$ Their findings set forth that FT3 can be a prognostic marker and it could also assist in stratifying severely ill COVID-19 cases for better management as low FT3 
independently predicts all-cause mortality. Nonetheless, the low T3 syndrome is a defense mechanism that the body puts in place during the acute phase which apparently does not require thyroid hormone replacement with levothyroxine.

Moreover, analysis of serum biomarkers in a longitudinal study revealed that COVID-19 patients with thyroid disease exhibited interminably relatively higher levels of the inflammatory response and cardiac injury biomarkers. COVID-19 subjects bearing thyroid disease were identified to progress into a critical subtype of the disease, significantly higher fatality rate $(P<0.0001)$ and lengthier hospital stay ( $>28$ days) $(P=0.048)$ compared with those without thyroid disease suggesting that thyroid disease could be linked to an unfavorable prognosis. ${ }^{78}$ In line with this, $\sim 15 \%$ of 191 patients presenting mild-to-moderate COVID-19 disease showed thyroid dysfunction while a low FT3 was significantly associated with systemic inflammation, suggesting a role in prognostic significance. ${ }^{26}$ This is also due to the SARS-CoV-2mediated direct impact on thyroid activity could result in the aggravation of preceding-thyroid disease (autoimmune). Apart from this, the euthyroid sick syndrome has also been displayed to be significantly associated with inflammatory markers. ${ }^{72}$ Contrary, recent research from the UK reported only a mild reduction in TSH and FT4 in the COVID-19 patients while the majority of them have exhibited euthyroidism. ${ }^{79}$ Similarly, a group from the United States found no correlation between hypothyroidism patients and COVID-19. ${ }^{80}$ These findings propose further research into the probable corollaries of the virus on the thyroid as the obtained results are inconclusive as of now.

Besides, the SARS-CoV-2 could also cause hyperactivation of the immune response through different T-cell lymphocytes (Th1/Th17) that results in activation and release of various pro-inflammatory cytokines including interleukins (IL1-IL6) and tumor necrosis factor $\alpha$ $(\mathrm{TNF} \alpha)$, and this process collectively induces "cytokine storm". ${ }^{81}$ In particular, the IL6 was elevated in the acute phase suggesting that it can lead to thyrotoxicosis as this condition is highly associated with IL6 elevation. ${ }^{82}$ Moreover, any alteration in the thyroid hormone transport proteins, and due to structural and biochemical alterations, altered TSH-secreting pituitary cells may result in aberrant biochemical thyroid profiles, mainly the "low triiodothyronine (T3) syndrome" and "euthyroid sick syndrome" wherein, low free triiodothyronine (FT3) has been shown to be associated with enhanced IL6 while the free thyroxine (FT4) is normal/fairly decreased and TSH is normal/ low. ${ }^{68}$ Generally, these anomalies reported in thyroid parameters of non-thyroid-related conditions are transient which are not normally therapeutically intervened. ${ }^{69}$ Still, the post-cytokine storm could cause the deregulation of the immune system that may result in diverse autoimmune thyroid disorders. ${ }^{83}$

\section{Thyroid Dysfunctions Also in Post- COVID Cases}

In addition to a prominent association between SARSCoV-2 and thyroid disease during the course or the acute phase of COVID-19, recent reports observed various thyroid diseases associated with the post-COVID-19 phase, and these are discussed in the following topics.

\section{SARS-CoV-2 Induces Subacute Thyroiditis in Post-COVID- 19 Patients}

\section{Subacute Thyroiditis: A Brief Note}

Subacute thyroiditis - painful inflammation of the thyroid gland. This transient state is self-limited. ${ }^{84}$ It has 4 phases and usually lasts 4-6 months. The initial phase of thyrotoxicosis results in acute thyroid pain and tends to go for 3-6 weeks or even longer and ultrasonography could be adapted for the differential diagnosis of thyrotoxicosis as this tool is non-invasive and cost-effective. ${ }^{85}$ The next phase is transient asymptomatic euthyroidism. The third phase is hypothyroidism and this lasts for some weeks to months and can even be permanent in $\sim 5 \%$. The end phase is the convalescence phase. Across this period thyroid gland functions become normal.

Subacute thyroiditis is mostly engendered by an infection of the virus. It is mainly anteceded by a primary respiratory infection. Various viruses are linked with subacute thyroiditis, for example, coxsackievirus, cytomegalovirus (CMV), echovirus, adenovirus, enterovirus, Epstein-Barr virus (EBV), influenza, mumps, and parvovirus B19. ${ }^{86}$ Women show three to five times more susceptibility than men between the age of 30-50 years. Moreover, this subacute thyroiditis is topographical and seasonal. This increasingly occurs in the summertime and autumn seasons. ${ }^{87}$

This disorder clinically presents the inception of intense pain in the area of the thyroid gland and exacerbates when the head is turned or swallowed and usually 
radiates to other nearby parts. ${ }^{88}$ In general, a significantly elevated erythrocyte sedimentation rate (ESR) is seen and hypermetabolism can be detected. Mainly, the thyroid has been reported to be solid, nodule-like, and remarkably delicate. The number of white blood cells (WBCs) has generally been detected within the normal range or marginally increased. More than $50 \%$ of patients represented thyrotoxicosis in the most acute phase and T4 was highly enhanced compared with T3 while TSH levels were very low or undetectable. The thyroglobulin level is high and radioactive iodine uptake (RAIU) is markedly low and mostly less than $2 \%$ at $24 \mathrm{hrs}^{84}$

The ATDs are not prescribed as subacute thyroiditis is a disorder that is caused by a virus that results in the release of preformed thyroid hormone but not new T3 and T4 synthesis. Beta-blockers are mainly used as therapeutic options to relieve the symptoms of this condition. In mild cases, nonsteroidal anti-inflammatory (NSAIDs) are administered to reduce inflammation and pain. Prednisone $(20-40 \mathrm{mg} /$ day $)$ is recommended for patients with severe pain for more than $2-4$ weeks. ${ }^{88}$ Twenty percent of these patients feel thyroid pain if they discontinue prednisone. ${ }^{84}$ RAIU could help the physician whether the patients are at higher risk for relapse indicating that low-level uptake of RAIU could display ongoing inflammation and recommendation for continuing steroid therapy. ${ }^{88}$

\section{Subacute Thyroiditis in Post-COVID-19 Cases}

Several viruses were known to cause subacute thyroiditis, whether SARS-CoV-2 has any association with thyroid was completely unknown until the first report came from Brancatella et al in 2020. The group showed for the first time that the SARS-CoV-2 infection could cause subacute thyroiditis. ${ }^{89}$ Following this initial observation, many other studies also reported the subacute thyroiditis associated with SARS-CoV-2. ${ }^{86,90-111}$ The cases were confirmed twice with the RT-PCR mostly using the nose/ throat swabs. Further, analyses of viral panels for the respiratory system showed negative results in these patients. Various sets of urine and blood cultures showed no other viral infection. Apart from these, hepatitis (HBV), Brucella, and human immunodeficiency virus (HIV) screening were negative. These patients presented with normal chest X-rays and computed tomography (CT). As summarized in Table 1, SARS-CoV-2 infection-induced subacute thyroiditis cases were in the age range of 18 to 69 , and the majority of them were women $(73.6 \%, 28 / 38)$. The onset of symptoms and diagnosis of subacute thyroiditis from the SARS-CoV-2 infection ranged from 3 days to 49 days. Of 38 subacute thyroiditis cases, 21 cases were detected in post-COVID-19 condition, 7 cases were identified during the acute phase, 5 cases were found at the end of the acute phase, and no information was available for the remaining 5 cases. All the patients had a high fever, palpitations with neck pain except one case which showed only neck pain and palpitation. Enhanced ESR and C-reactive protein (CRP) were predominantly detected. Majority of the cases presented with higher levels of FT4 and lower to untraceable levels of TSH suggesting overt thyrotoxicosis in the acute phase. In some cases, the ratio of FT3/FT4 was low with elevated thyroglobulin (Tg) level reflecting the consequence of the destructive process. Except for a few cases, TSH receptor antibodies (TSHR $\mathrm{Ab})$ were mostly absent. The thyroid peroxidase (TPOAb) and thyroglobulin antibodies ( $\mathrm{TgAb}$ ) were positive in some cases yet within the normal range. Ultrasound examination of the thyroid displayed a relative heterogeneous diffuse decrease of vascularity and parenchyma. These cases showed a lower level or absence of radionuclide tracer uptake. Overall, the thyroid profile, inflammatory markers, and blood parameters of the COVID-19 cases reflected subacute thyroiditis and were managed by antiinflammatory treatments with the NSAID or prednisone (16-25 mg/day) for 4 weeks and progressively tapered. The patients with anxiety, palpitations, or tremor were mostly treated with a beta-blocker, propranolol (40-120 $\mathrm{mg}$ ) while thionamides were avoided. Patients with concomitant COVID-19 and subacute thyroiditis were administered with hydroxychloroquine $200 \mathrm{mg}$ bid for 5 days in addition to prednisone. ${ }^{90}$ The time period from the diagnosis of subacute thyroiditis to recovery ranged from 2 to 4 weeks. As displayed in Figure 3, subacute thyroiditis is the probable basis of direct viral infection and/or effect of viral protein-induced "cytokine storm".

\section{SARS-CoV-2 Causes Graves' Disease}

An autoimmune disease wherein immune cells attack the thyroid which drives the production of an excess hormone that the body normally requires that yields hyperthyroidism. This is more frequently observed in middle-aged women. Recent pandemic COVID-19 was shown to trigger the immune system suggesting that SARS-CoV-2 could hyperactivate latent or new-onset autoimmunity. 


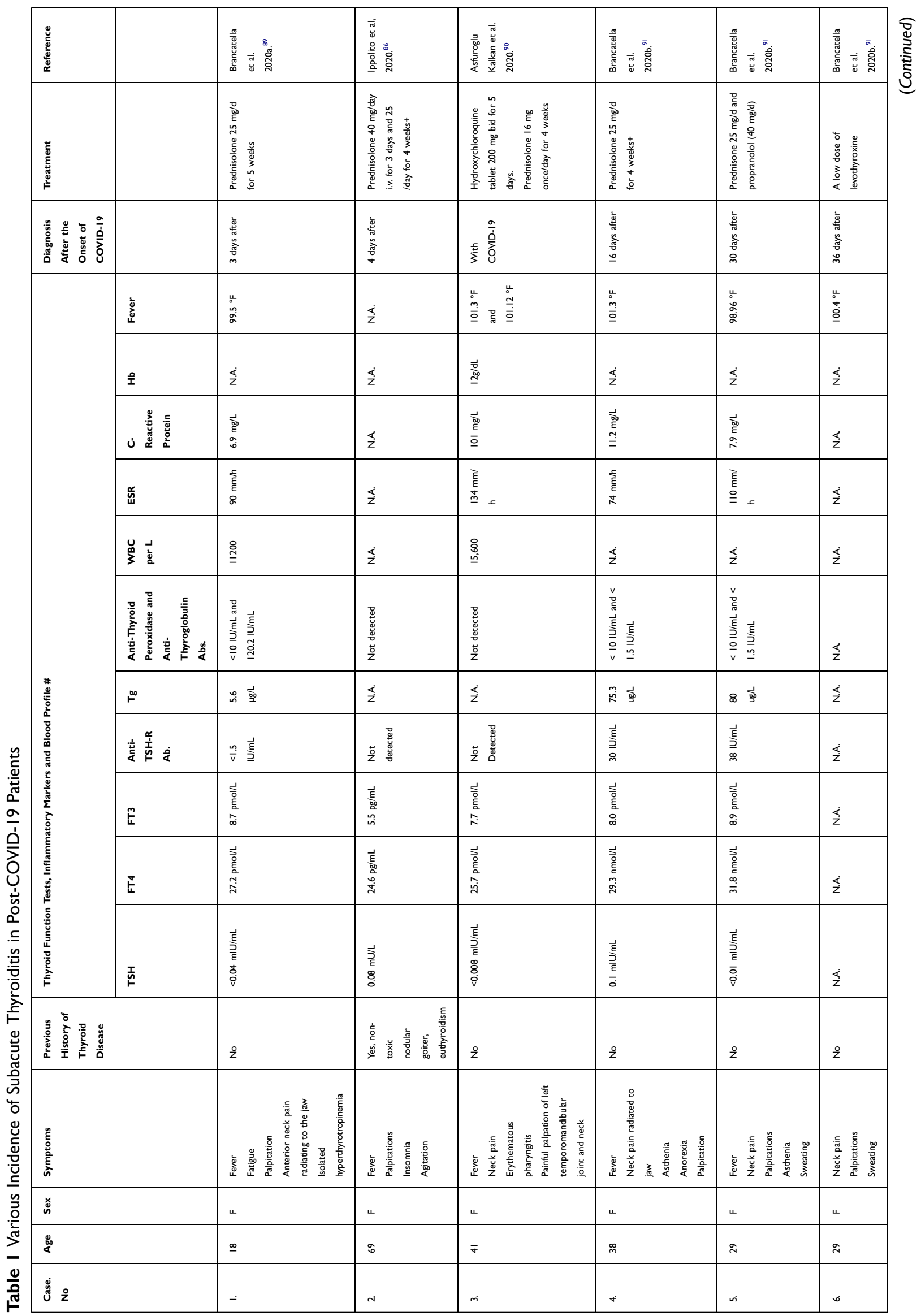




\begin{tabular}{|c|c|c|c|c|c|c|c|c|}
\hline 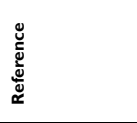 & & 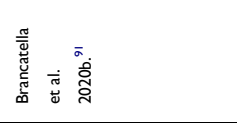 & 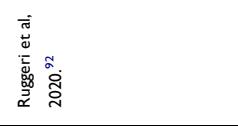 & 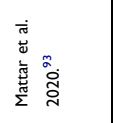 & 惡 & 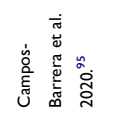 & 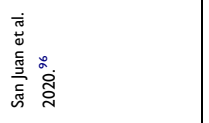 & 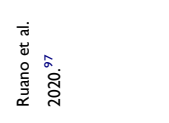 \\
\hline 䓂 & & 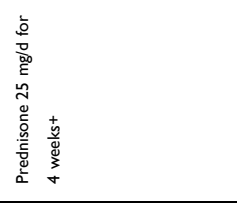 & 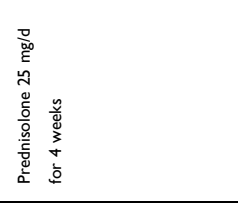 & 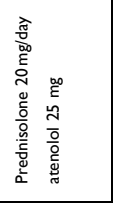 & 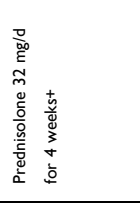 & $\frac{\pi}{z}$ & 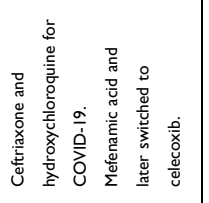 & 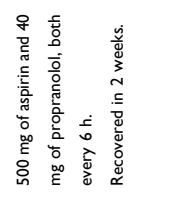 \\
\hline 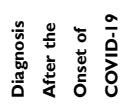 & & $\begin{array}{l}\text { 高 } \\
\text { 旁 } \\
\text { 竞 } \\
\text { i }\end{array}$ & 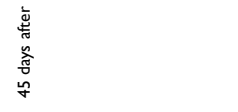 & 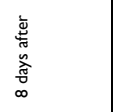 & 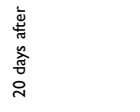 & 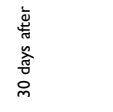 & 产 & 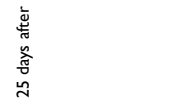 \\
\hline \multirow{11}{*}{ 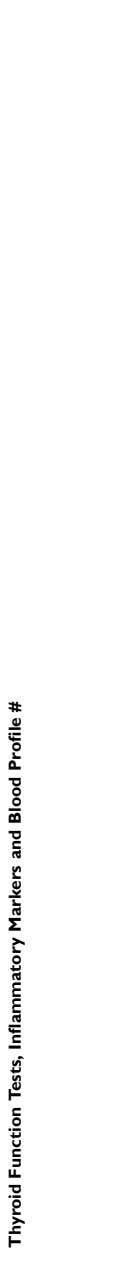 } & 高 & 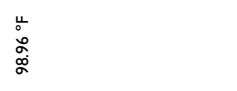 & \begin{tabular}{l}
\multicolumn{4}{c}{} \\
$\vdots$ \\
$\vdots$ \\
$\alpha$ \\
$\alpha$
\end{tabular} & $\begin{array}{l}\stackrel{u}{u} \\
\stackrel{0}{\alpha} \\
\alpha \\
\alpha\end{array}$ & 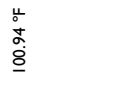 & 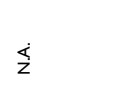 & 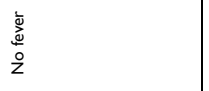 & $\begin{array}{l}\stackrel{u}{0} \\
\stackrel{m}{\bar{\theta}}\end{array}$ \\
\hline & $\stackrel{0}{I}$ & $\stackrel{\pi}{z}$ & $\stackrel{\pi}{z}$ & 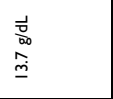 & 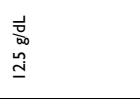 & 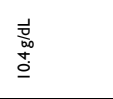 & $\stackrel{\pi}{z}$ & $\stackrel{\mathbb{s}}{z}$ \\
\hline & ن & 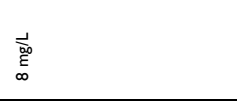 & 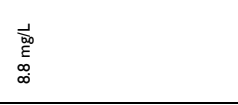 & 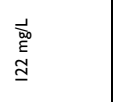 & 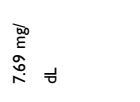 & 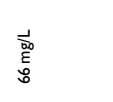 & 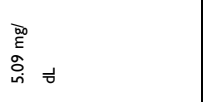 & $\begin{array}{l}\overrightarrow{\underline{w}} \\
\underline{\underline{E}} \\
\underline{\underline{E}}\end{array}$ \\
\hline & 总 & $\stackrel{\pi}{z}$ & 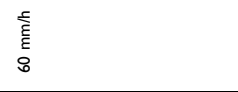 & $\overleftrightarrow{\dot{z}}$ & 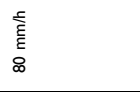 & $\stackrel{\underline{\underline{E}}}{\approx}=$ & $\overleftrightarrow{\mathbb{Z}}$ & $\begin{array}{l}\text { 裹 } \\
\stackrel{\underline{o}}{=}\end{array}$ \\
\hline & 愛 & $\frac{\Delta}{z}$ & 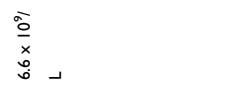 & $\stackrel{\substack{0 \\
=}}{=}$ & $\stackrel{8}{=}$ & $\begin{array}{l}\overline{\bar{s}} \\
\overline{\frac{5}{2}}\end{array}$ & 䙴 & $\stackrel{x}{\stackrel{x}{=}} \stackrel{\vec{\partial}}{\underline{\partial}}$ \\
\hline & 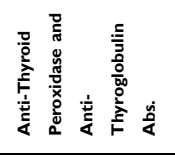 & $\stackrel{\Delta}{z}$ & 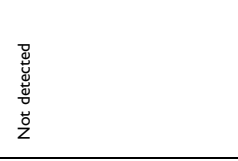 & 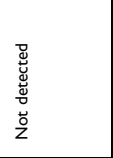 & 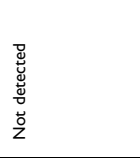 & 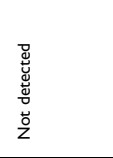 & 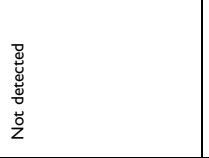 & 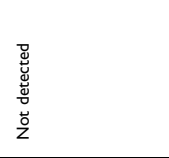 \\
\hline & $\stackrel{\infty}{\llcorner}$ & $\frac{s}{z}$ & 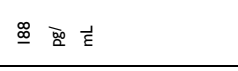 & $\frac{1}{z}$ & $\stackrel{s}{z}$ & $\frac{s}{z}$ & $\frac{1}{z}$ & $\frac{s}{z}$ \\
\hline & 毫喜㸃 & 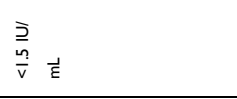 & 菎 & 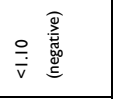 & 蒙 & $\stackrel{\pi}{z}$ & 萼总 & 蒙 \\
\hline & $F$ & 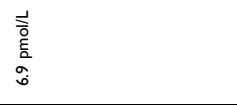 & لُ & 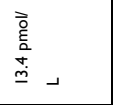 & 离 & $\frac{d}{z}$ & $\frac{d}{z}$ & $\frac{\pi}{z}$ \\
\hline & $\vec{E}$ & 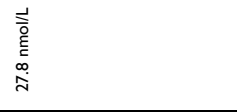 & 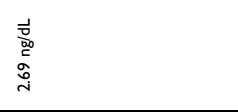 & 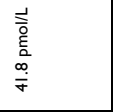 & 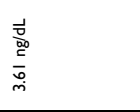 & 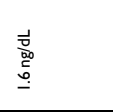 & $\begin{array}{l}\overrightarrow{\mathrm{E}} \\
\text { 言 } \\
\text { o. } \\
\underline{-}\end{array}$ & 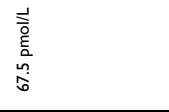 \\
\hline & $\stackrel{I}{h}$ & 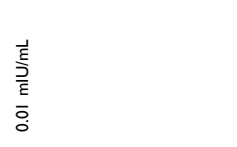 & 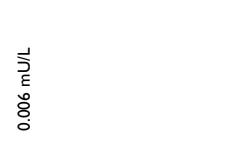 & $\begin{array}{l}\vec{J} \\
\stackrel{\vec{E}}{E} \\
\stackrel{\partial}{\dot{Q}}\end{array}$ & 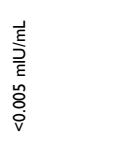 & 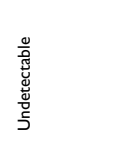 & 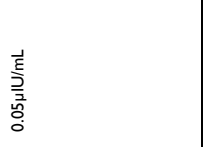 & 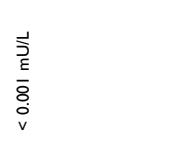 \\
\hline \multicolumn{2}{|l|}{ 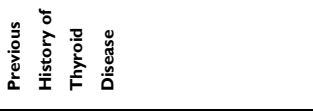 } & $\stackrel{\circ}{z}$ & $\stackrel{\circ}{z}$ & $\stackrel{\circ}{z}$ & $\stackrel{\circ}{z}$ & & $\stackrel{2}{z}$ & $\stackrel{\circ}{z}$ \\
\hline 高 & & 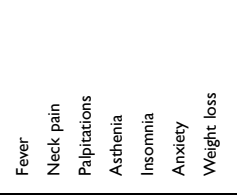 & 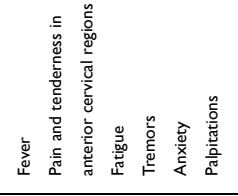 & 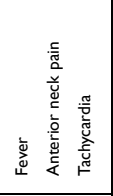 & 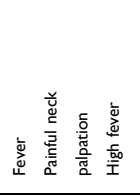 & 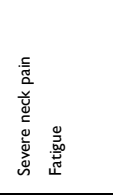 & 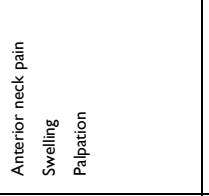 & 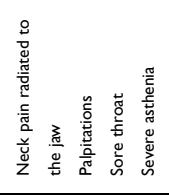 \\
\hline ○̊ & & 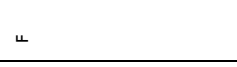 & ${ }^{\Perp}$ & $\Sigma$ & $\Sigma$ & ${ }_{4}$ & ${ }^{4}$ & ${ }_{4}$ \\
\hline 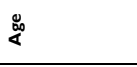 & & 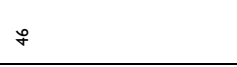 & $q$ & $\Phi$ & \& & $\hat{m}$ & F & $\infty$ \\
\hline نُ & & $r$ & $\infty$ & $\alpha$ & $\stackrel{\circ}{9}$ & $\dot{=}$ & $\simeq$ & $\underline{m}$ \\
\hline
\end{tabular}




\begin{tabular}{|c|c|c|c|c|c|c|}
\hline 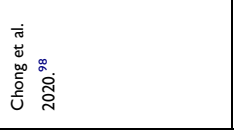 & 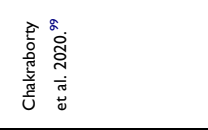 & 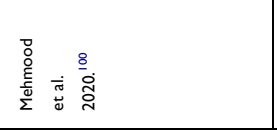 & 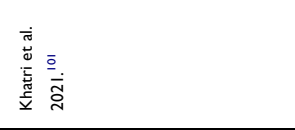 & 言高就 & 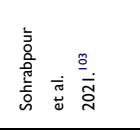 & 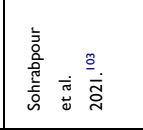 \\
\hline 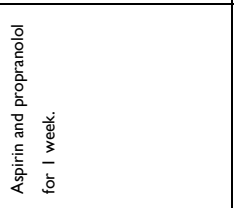 & 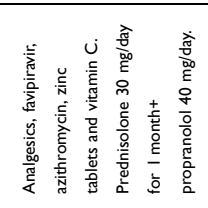 & 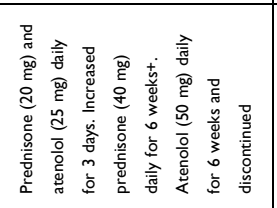 & 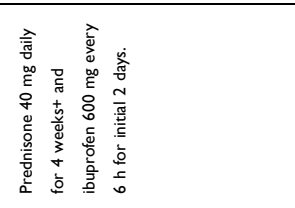 & 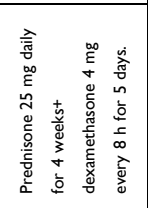 & 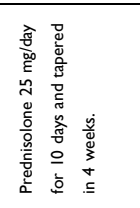 & 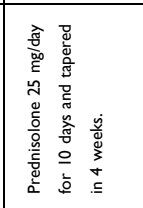 \\
\hline 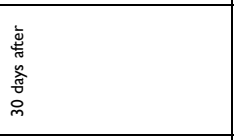 & 产产 & 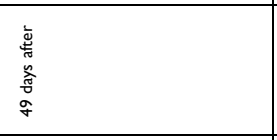 & 辤 & $\begin{array}{l}\frac{2}{\hat{y}} \\
\frac{\bar{x}}{x} \\
\end{array}$ & 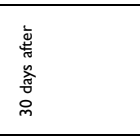 & 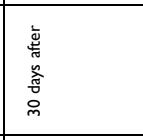 \\
\hline 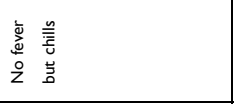 & 䛓 & 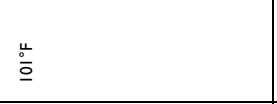 & 営 & 尙 & 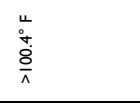 & \begin{tabular}{|l} 
\\
产 \\
$\frac{1}{n}$ \\
\end{tabular} \\
\hline 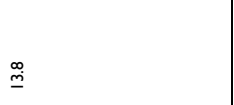 & 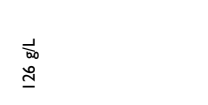 & $\frac{1}{2}$ & $\frac{\overrightarrow{\mathrm{w}}}{\mathrm{a}}$ & 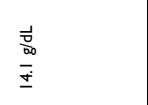 & $\frac{d}{2}$ & $\frac{1}{2}$ \\
\hline 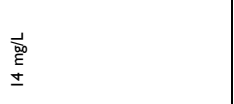 & 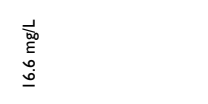 & 峷 & 熟 & 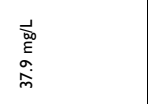 & 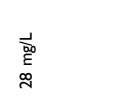 & 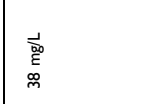 \\
\hline 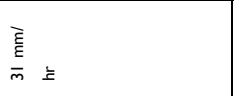 & 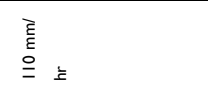 & 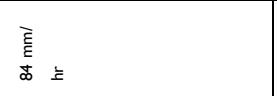 & 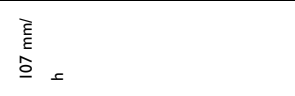 & 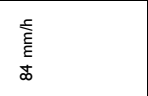 & 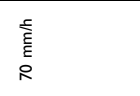 & 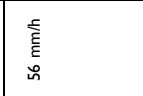 \\
\hline 竞 & 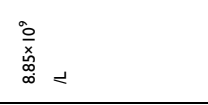 & $\frac{\overline{\bar{s}}}{2}$ & 希表旁 & $\frac{\bar{s}}{\bar{z}}$ & 总 & 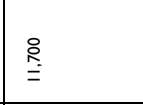 \\
\hline$\underline{\underline{\underline{\underline{I}}}}$ & $\frac{1}{2}$ & 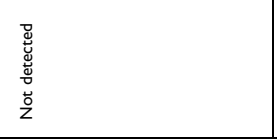 & 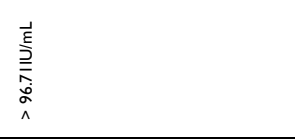 & 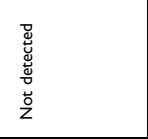 & $\frac{d}{z}$ & $\frac{1}{2}$ \\
\hline$\frac{d}{2}$ & $\frac{1}{2}$ & $\frac{\pi}{z}$ & $\frac{\pi}{z}$ & $\frac{\pi}{z}$ & $\frac{d}{z}$ & $\frac{1}{2}$ \\
\hline$\frac{\pi}{z}$ & $\frac{1}{2}$ & $\frac{\pi}{2}$ & $\stackrel{\varrho}{\bar{y}}$ & 雚 & $\frac{\pi}{2}$ & $\frac{1}{2}$ \\
\hline$\frac{1}{2}$ & $\frac{s}{2}$ & $\frac{s}{2}$ & 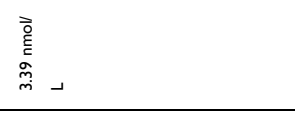 & $\frac{s}{2}$ & 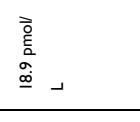 & 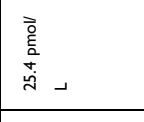 \\
\hline 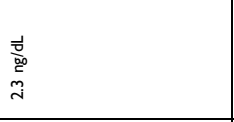 & $\frac{1}{z}$ & 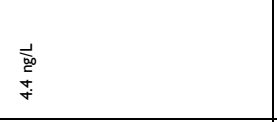 & 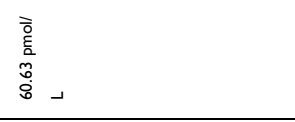 & $\frac{\pi}{2}$ & 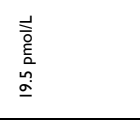 & 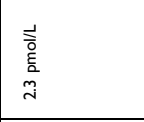 \\
\hline 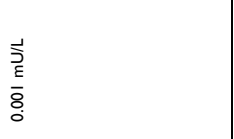 & $\frac{\mathrm{g}}{\mathrm{g}}$ & 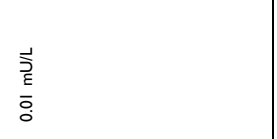 & $\begin{array}{l}\frac{\vec{z}}{\underline{\underline{z}}} \\
\frac{\partial}{\partial} \\
\text { v }\end{array}$ & 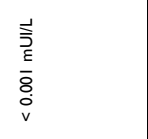 & 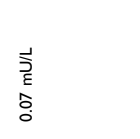 & 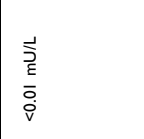 \\
\hline$\stackrel{2}{2}$ & $\stackrel{0}{2}$ & $\stackrel{\circ}{z}$ & $\stackrel{\circ}{z}$ & $\stackrel{2}{z}$ & $\frac{\pi}{2}$ & $\frac{1}{2}$ \\
\hline 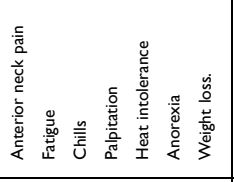 & 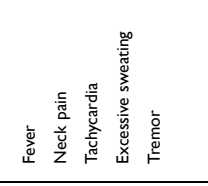 & 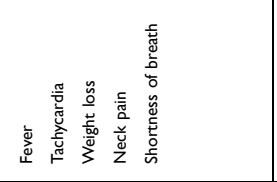 & 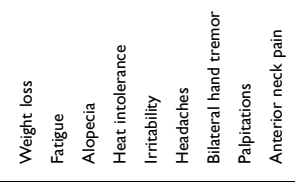 & 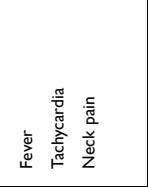 & 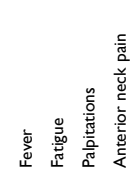 & 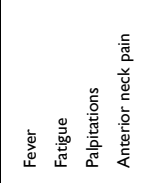 \\
\hline$\Sigma$ & $\Sigma$ & ${ }_{4}$ & ${ }_{4}$ & $\Sigma$ & ${ }_{4}$ & ـ \\
\hline$\hat{m}$ & $\infty$ & $\therefore$ & $\bar{F}$ & $\infty$ & $\therefore$ & $\approx$ \\
\hline \pm & $\underline{\underline{n}}$ & $\simeq$ & $=$ & $\stackrel{\infty}{ }$ & 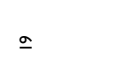 & $\therefore$ \\
\hline
\end{tabular}




\begin{tabular}{|c|c|c|c|c|c|c|c|c|c|}
\hline 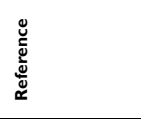 & & 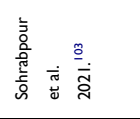 & 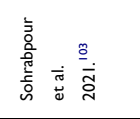 & 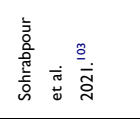 & 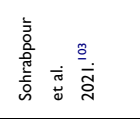 & 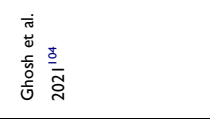 & 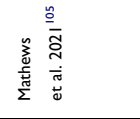 & 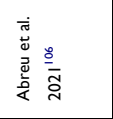 & 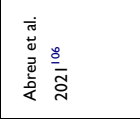 \\
\hline 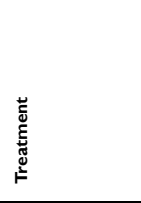 & & 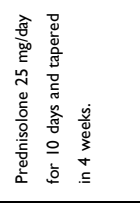 & 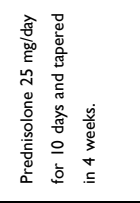 & 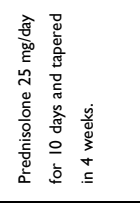 & 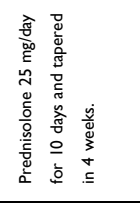 & 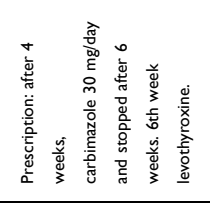 & 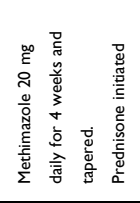 & 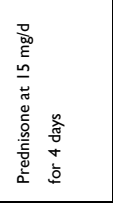 & 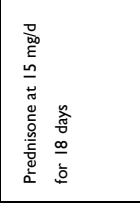 \\
\hline 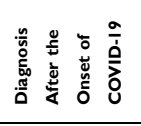 & & 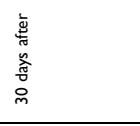 & $\begin{array}{l}\text { 离 } \\
\text { 旁 } \\
\text { 咅 } \\
\text { 总 } \\
\end{array}$ & 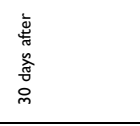 & 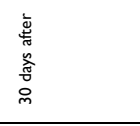 & 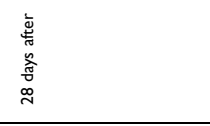 & 产产 & 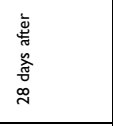 & 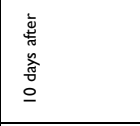 \\
\hline \multirow{11}{*}{ 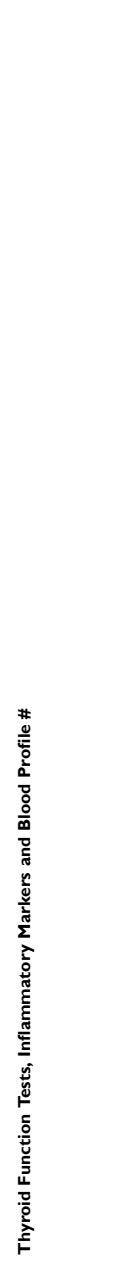 } & \begin{tabular}{|l} 
\\
\end{tabular} & 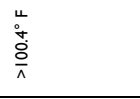 & 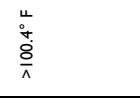 & 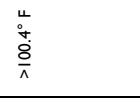 & 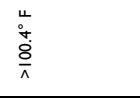 & $\begin{array}{l}\stackrel{u}{a} \\
\stackrel{\Xi}{\Xi} \\
\end{array}$ & 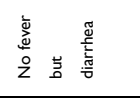 & $\stackrel{\stackrel{\Delta}{\circ}}{\underline{\Delta}}$ & $\overleftrightarrow{Z}$ \\
\hline & 옹 & $\frac{\pi}{z}$ & $\stackrel{\mathbb{x}}{z}$ & $\stackrel{\pi}{z}$ & $\frac{1}{2}$ & $\frac{4}{2}$ & $\frac{\pi}{z}$ & $\frac{\pi}{2}$ & $\frac{\pi}{z}$ \\
\hline & 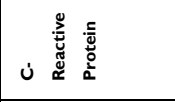 & 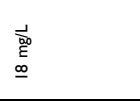 & 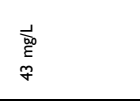 & $\begin{array}{l}\frac{\vec{t}}{\mathrm{w}} \\
\frac{\mathrm{E}}{\mathrm{n}} \\
\mathrm{n}\end{array}$ & 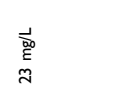 & 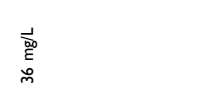 & 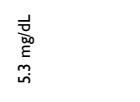 & $\frac{s}{z}$ & $\mathbb{Z}$ \\
\hline & 总 & 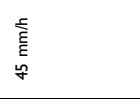 & 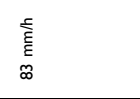 & 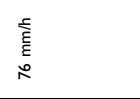 & 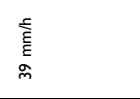 & 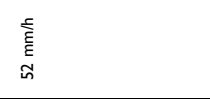 & 离 & $\frac{\pi}{z}$ & $\stackrel{\mathbb{S}}{z}$ \\
\hline & 尙 & ఫ్ఫે & 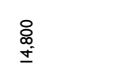 & $\stackrel{\circ}{\stackrel{\circ}{\complement}}$ & 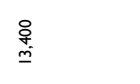 & $\stackrel{\circ}{\stackrel{\circ}{9}}$ & $\frac{s}{z}$ & $\frac{d}{z}$ & $\stackrel{\mathbb{Z}}{Z}$ \\
\hline & 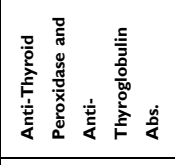 & $\frac{\pi}{z}$ & $\stackrel{\mathbb{Z}}{z}$ & $\frac{4}{2}$ & $\stackrel{i}{\mathbf{z}}$ & $\frac{1}{z}$ & $\frac{\pi}{z}$ & $\frac{\pi}{z}$ & $\stackrel{\Delta}{Z}$ \\
\hline & $\stackrel{\infty}{\circ}$ & $\frac{\dot{s}}{z}$ & $\frac{\dot{s}}{z}$ & $\frac{d}{2}$ & $\frac{\pi}{2}$ & $\frac{\dot{s}}{z}$ & $\frac{\pi}{z}$ & $\frac{d}{2}$ & $\stackrel{\Delta}{z}$ \\
\hline & 竞盖运 & $\stackrel{s}{z}$ & $\stackrel{\mathbb{S}}{z}$ & $\stackrel{\$}{z}$ & $\frac{\pi}{2}$ & $\stackrel{\pi}{z}$ & $\frac{\pi}{z}$ & $\frac{4}{2}$ & $\mathbb{Z}$ \\
\hline & $\tilde{E}$ & 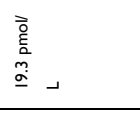 & 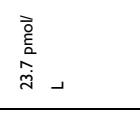 & 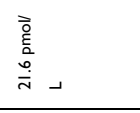 & $\begin{array}{l}\overline{\overline{\bar{c}}} \\
\text { 产 } \\
\underline{\underline{\alpha}}\end{array}$ & $\frac{s}{z}$ & $\frac{\pi}{z}$ & $\frac{1}{2}$ & 这 \\
\hline & $E$ & 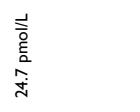 & 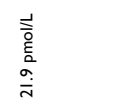 & 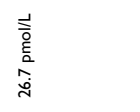 & 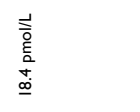 & $\stackrel{\mathbb{Z}}{z}$ & 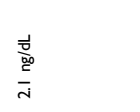 & $\frac{4}{z}$ & 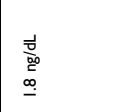 \\
\hline & 甹 & 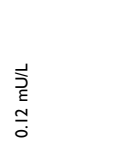 & $\begin{array}{l}\vec{J} \\
\underline{\underline{E}} \\
\overline{\dot{O}} \\
\mathrm{v}\end{array}$ & $\begin{array}{l}\vec{J} \\
\underline{E} \\
\stackrel{5}{0}\end{array}$ & 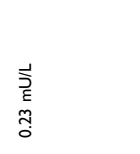 & 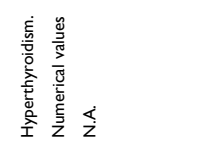 & 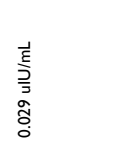 & $\stackrel{\Delta}{z}$ & $\overleftrightarrow{\mathbb{Z}}$ \\
\hline 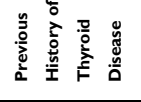 & & $\frac{s}{z}$ & $\stackrel{\mathbb{S}}{z}$ & $\frac{\pi}{2}$ & $\frac{1}{2}$ & z̊ & $\stackrel{\circ}{z}$ & $\frac{\pi}{2}$ & 울 \\
\hline 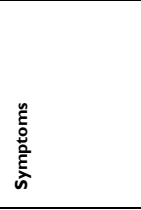 & & 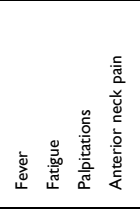 & 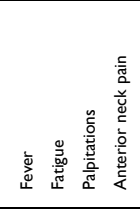 & 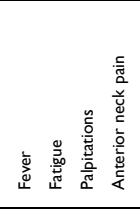 & 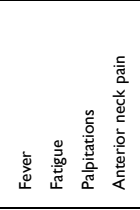 & 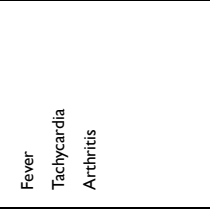 & 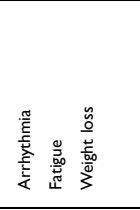 & 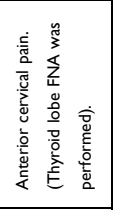 & 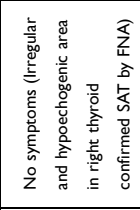 \\
\hline ڤ̊ & & $\Sigma$ & 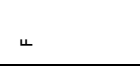 & $\Sigma$ & ${ }^{\Perp}$ & $\Sigma$ & $\Sigma$ & ${ }_{\Perp}$ & 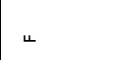 \\
\hline 迎 & & $\ddot{m}$ & $\bar{F}$ & $\approx$ & \pm & in & 6 & $\Phi$ & ळ \\
\hline :ٌّ & & $\bar{a}$ & $\approx$ & $\approx$ & $\dot{\sim}$ & 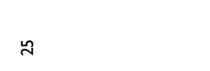 & $\stackrel{\sim}{\sim}$ & $\hat{\sim}$ & $\stackrel{\infty}{\infty}$ \\
\hline
\end{tabular}




\begin{tabular}{|c|c|c|c|c|c|c|c|c|c|}
\hline 焉 & 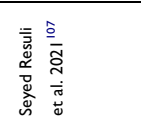 & 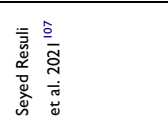 & 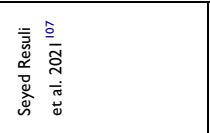 & 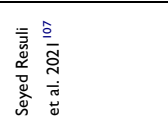 & 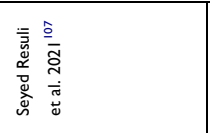 & 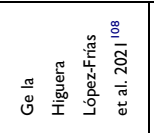 & 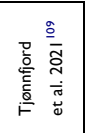 & 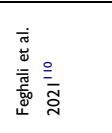 & 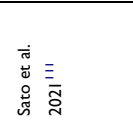 \\
\hline$\frac{\pi}{z}$ & 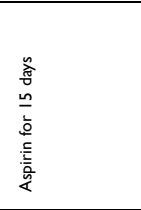 & 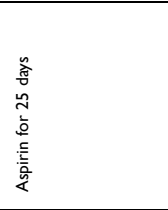 & 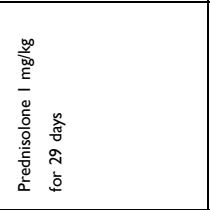 & 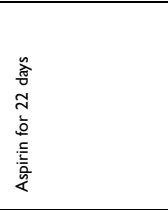 & 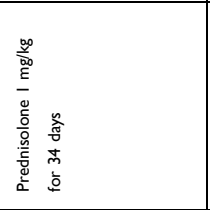 & 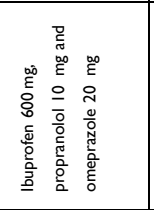 & 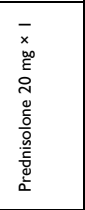 & 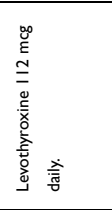 & 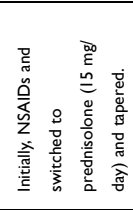 \\
\hline 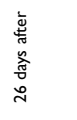 & $\mathbb{z}$ & $\underline{\mathbb{z}}$ & 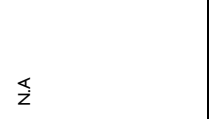 & $\stackrel{\mathbb{Z}}{z}$ & $\stackrel{\pi}{z}$ & 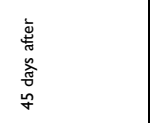 & 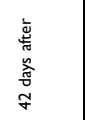 & 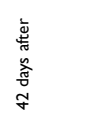 & 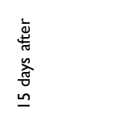 \\
\hline$\frac{\underline{\nu}}{\bar{\Sigma}}$ & $\stackrel{\mathbb{z}}{z}$ & $\frac{\pi}{z}$ & $\stackrel{\mathbb{z}}{z}$ & $\stackrel{\pi}{z}$ & $\underline{\pi}$ & 咅总哀 & $\begin{array}{l}\stackrel{u}{\circ} \\
\vdots \\
\alpha \\
\alpha\end{array}$ & $\frac{D}{\bar{\nu}}$ & 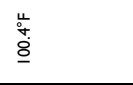 \\
\hline$\frac{1}{z}$ & $\stackrel{\mathbb{z}}{z}$ & $\frac{\pi}{z}$ & 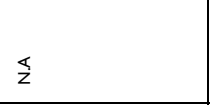 & $\stackrel{\pi}{z}$ & $\frac{\pi}{z}$ & $\stackrel{\mathbb{S}}{z}$ & $\frac{\pi}{2}$ & $\frac{\pi}{z}$ & 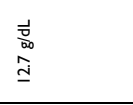 \\
\hline$\$$ & $\frac{\pi}{z}$ & $\frac{\pi}{z}$ & $\stackrel{s}{z}$ & $\frac{\pi}{z}$ & $\frac{\pi}{z}$ & 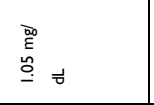 & ฌ & $\frac{\pi}{z}$ & 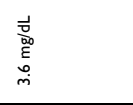 \\
\hline$\frac{\pi}{z}$ & 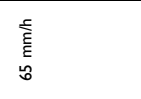 & 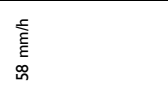 & 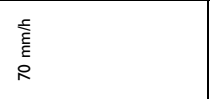 & 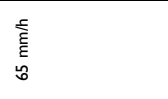 & 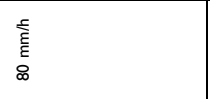 & 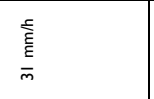 & 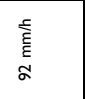 & $\frac{\pi}{2}$ & 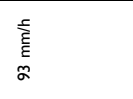 \\
\hline$\frac{s}{z}$ & $\frac{\pi}{z}$ & $\frac{\pi}{z}$ & $\stackrel{s}{z}$ & $\frac{\pi}{z}$ & $\frac{\pi}{z}$ & 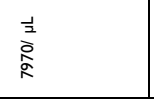 & $\begin{array}{l}x \\
\stackrel{x}{=} \\
\stackrel{0}{=}\end{array}$ & $\frac{1}{z}$ & $\begin{array}{l}\frac{\partial}{x} \\
\underline{x} \\
\underline{8}\end{array}$ \\
\hline$\frac{\pi}{z}$ & 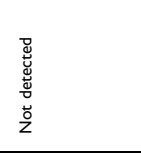 & 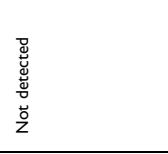 & 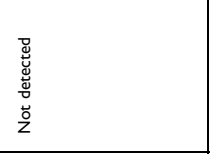 & 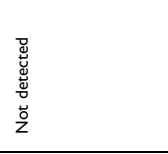 & 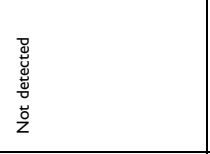 & 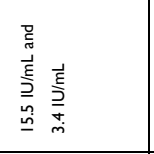 & v & $\begin{array}{l}\overrightarrow{\underline{E}} \\
\frac{\vec{z}}{3} \\
\end{array}$ & 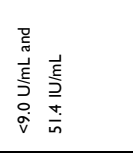 \\
\hline$\frac{\pi}{2}$ & $\stackrel{s}{z}$ & $\frac{\pi}{z}$ & $\frac{\pi}{z}$ & $\frac{\pi}{z}$ & $\frac{\pi}{z}$ & $\frac{d}{z}$ & $\stackrel{\Phi}{z}$ & $\frac{d}{2}$ & $\frac{\$}{z}$ \\
\hline$\frac{\pi}{2}$ & 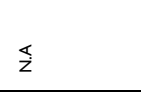 & $\stackrel{\mathbb{s}}{z}$ & $\stackrel{\mathbb{z}}{z}$ & $\stackrel{\mathbb{1}}{z}$ & $\frac{\pi}{z}$ & ì & $\dot{\vec{v}}$ & $\frac{\pi}{z}$ & $\stackrel{\circ}{\grave{i}}$ \\
\hline$\frac{d}{z}$ & $\frac{\dot{z}}{z}$ & 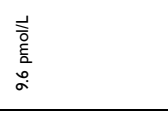 & 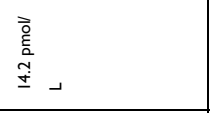 & $\begin{array}{l}\overline{\bar{c}} \\
\stackrel{\overline{⿳ 亠 口}}{=} \\
=\end{array}$ & 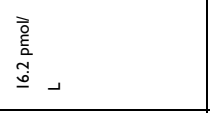 & 竞高 & 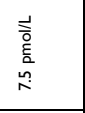 & $\frac{1}{z}$ & 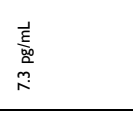 \\
\hline$\stackrel{\mathbb{s}}{z}$ & 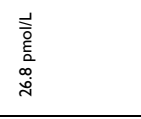 & 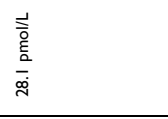 & $\begin{array}{l}\frac{\overrightarrow{\bar{c}}}{\overline{\underline{\underline{a}}}} \\
\frac{\bar{m}}{\dot{\gamma}} \\
\end{array}$ & 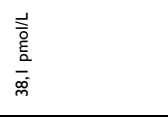 & 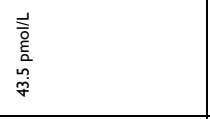 & 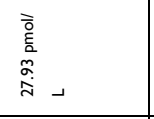 & 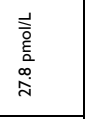 & 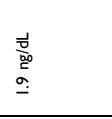 & 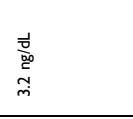 \\
\hline$\stackrel{\Delta}{z}$ & $\frac{1}{2}$ & $\stackrel{\pi}{z}$ & $\frac{\pi}{z}$ & $\stackrel{\mathbb{Z}}{z}$ & $\frac{\pi}{z}$ & 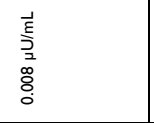 & $\begin{array}{l}\frac{\vec{J}}{\bar{\xi}} \\
\frac{\bar{\varepsilon}}{\partial}\end{array}$ & $\begin{array}{l}\frac{1}{\bar{z}} \\
\frac{\mathrm{E}}{\partial} \\
\end{array}$ & $\begin{array}{l}\overrightarrow{\underline{E}} \\
\frac{\partial}{\partial} \\
\stackrel{\bar{D}}{0}\end{array}$ \\
\hline$\frac{s}{2}$ & $\stackrel{2}{2}$ & $\stackrel{\circ}{2}$ & $\stackrel{\circ}{2}$ & $\stackrel{\circ}{z}$ & $\stackrel{\circ}{z}$ & $\stackrel{2}{z}$ & $\stackrel{2}{2}$ & $\frac{d}{2}$ & $\stackrel{\circ}{2}$ \\
\hline 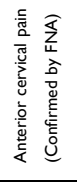 & 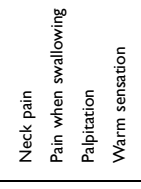 & 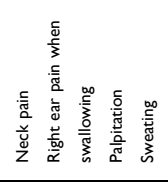 & 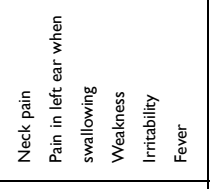 & 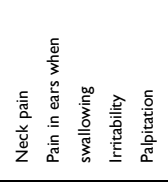 & 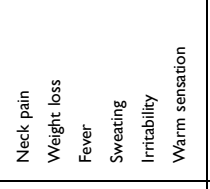 & 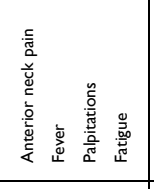 & 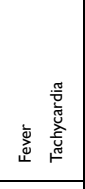 & 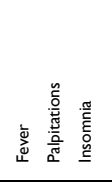 & 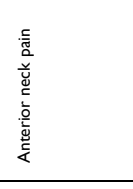 \\
\hline${ }^{\circ}$ & $\stackrel{ }{\perp}$ & 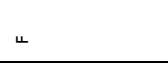 & 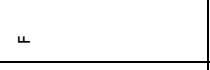 & 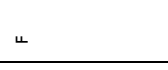 & 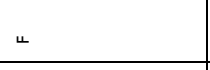 & $\stackrel{ }{\Perp}$ & $\Sigma$ & $\stackrel{ }{\perp}$ & $\stackrel{ }{\perp}$ \\
\hline$\stackrel{\text { }}{\circ}$ & $\approx$ & i & 7 & 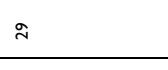 & $\bar{\sim}$ & $\stackrel{m}{\circ}$ & 8 & $\bar{\sigma}$ & $\bar{m}$ \\
\hline ते & 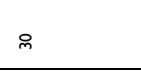 & $\bar{m}$ & $\tilde{m}$ & $\check{m}$ & $\stackrel{ \pm}{m}$ & $\stackrel{m}{m}$ & $\stackrel{\infty}{\circ}$ & in & $\stackrel{\infty}{\infty}$ \\
\hline
\end{tabular}




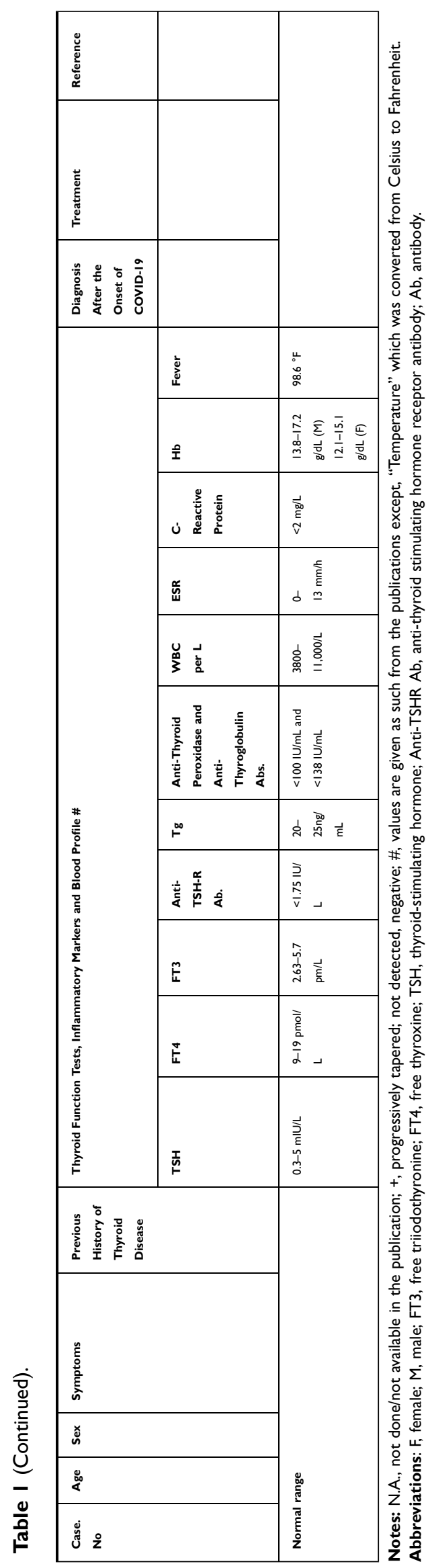

More recently, Mateu-Salat et al, for the first time, showed that SARS-COV-2 is a driver for Graves' disease in post-COVID-19 cases. ${ }^{112}$ Previous studies have demonstrated the presence of some virus-like particles in the follicular epithelium derived from subacute thyroiditis patients. ${ }^{64}$ Nonetheless, as of today, how the SARS-CoV2 attacks the thyroid remains completely unknown. The virus exploits ACE2 as a receptor for its initial cellular entry. It has been found that the ACE2 protein is highly expressed in the thyroid similar to the cardiac tissues, blood vessels and lungs thus enhance the chances of getting SARS-CoV-2 infection to the thyroid. ${ }^{3,4}$ Therefore, normally people have the risk of getting thyroid disease including subacute thyroiditis and Graves' disease in the post-COVID-19 scenario.

On the other hand, hyperthyroidism was shown to trigger the overexpression of the ACE2 due to vital regulation of the counter-regulatory components of RAAS (renin-angiotensin-aldosterone system), which also induces angiotensin 1-7 levels and Mas receptor. ${ }^{113}$ It suggests that people with thyroid disease, particularly hyperthyroidism, are a condition that could predispose the enhanced chances of acquiring SARS-CoV-2 infection while normal people readily get thyroid disease in the post-COVID-19 scenario.

As listed in Table 2, a study for the first time reported two cases of Graves' disease which was identified after SARS-CoV-2 infection. Of two, one case had a previous history of Graves' disease albeit she was free of disease for $>30$ years. ${ }^{112}$ Recently, another study also described two cases of Graves' disease in post-COVID-19 condition, and both the cases had a previous history of Graves' disease but they were free of disease for one year in one case and four years in another case. ${ }^{114}$ All the postCOVID-19 cases with Graves' disease showed the TSH of $<0.01 \mathrm{mIU} / \mathrm{mL}$ which was below the level of normal value, $0.3-5 \mathrm{mIU} / \mathrm{mL}$ suggesting severe hyperthyroidism. These cases were shown to be recovered after the conventional ATDs treatment. ${ }^{112,114-119}$

Environmental risk factors including viruses and stress are often implicated in autoimmune thyroid diseases. ${ }^{120}$ As depicted in Figure 3, SARS-CoV-2 infection-mediated hyperactivation of the pro-inflammatory conditions might reactivate Graves' disease via triggering an immunological cascade. Nevertheless, it remains not completely understood as only the inflammatory condition-associated immune response is partly studied in Graves' disease. Viral inflammatory condition is mainly induced by 
cytokines, Th1, and IL-6 while the pathogenesis of Graves' disease is seemingly regulated by Th2 autoimmune response. On the other hand, an enhanced amount of IL-6 was shown within the Graves' disease cases indicating differential and complex effects of cytokines via their cellular receptors. ${ }^{121}$ Consistently, T-cell subsets, Th17 and Th22 were markedly implicated in the diverse clinical course of the autoimmune-thyroid diseases (AITDs) including, Hashimoto thyroiditis and Graves' disease suggest that rewiring the role of the Th1-Th2 on the AITD pathogenesis. ${ }^{53,122}$

Furthermore, it is reasonable to suggest that hyperthyroid patients may have the paramount risk for SARS-CoV-2 infection and worst disease progression as these patients have hyperthyroidism-induced deregulated RAAS. This raises the possibility that susceptibility to the virus could be attributable to the cause of hyperthyroidism-induced overexpression of ACE2 or thyroid disease, particularly Graves' disease is the effect of viral pathogeneses. Consistently, the SARS-CoV-2-mediated thyrotoxic crisis was recently reported in a patient with a previous condition of Graves' disease. ${ }^{123}$ Moreover, this warrants the importance of obtaining a clear thyroid hormone profile in the initial days of viral infection or onset of COVID-19 in clinical settings and that would clearly define the preexisting thyroid condition as most SARS-CoV-2-induced thyroid diseases arise one-to-two months later the infection.

Contemplating pre-existing medical conditions mainly thyroid ailments, biochemical profile of thyroid, and optimal therapeutic plan may curtail the morbidity and mortality associated with SARS-CoV-2 and thyroid disease. Future studies are required to clarify "cause or effect", that would shed light on dark matter, SARS-CoV-2 and thyroid. As per recent guidelines, hyperthyroid patients with COVID-19 disease can be continued with anti-thyroid drug treatment regardless of their care in hospitals, special care centers for COVID-19, or home quarantine. Because uncontrolled hyperthyroidism could add to the severity of COVID-19 disease that may result in an unfavorable outcome. The drugs methimazole (MMI) and propylthiouracil (PTU) are recommended for oral administration of COVID-19 patients as intravenous solutions of MMI or PTU are scarcely available in the majority of the countries. ${ }^{124}$ This report once again reminds the physicians and/or researchers can be more conversant of the possible link between SARS-CoV-2 and Graves' disease, and other possible unidentified thyroid dysfunction which could fall under this umbrella.

\section{The SARS-CoV-2 Infection Causes Hashimoto's Thyroiditis}

Recently, the first case of Hashimoto's thyroiditis in the postCOVID-19 scenario was reported. ${ }^{83}$ A forty-five-year-old, non-smoking, gentleman was diagnosed with COVID-19 through an RT-PCR test from a nasopharyngeal swab. A week later, he recovered from the mild COVID-19 complications, yet possessed acute-onset of extreme fatigue with muscle feebleness without other related symptoms and he was not under any medication including supplements. He has no personal history of thyroid disorders. A careful check revealed that the man was not feverish, hemodynamically steady, exhibited normal electrolyte level with no anemia, cardiac, respiratory, abdominal, neurological issues, bradycardia, hypothermia, and the person was found to be clinically euthyroid without goiter. Further profiling of thyroid hormones revealed an enhanced TSH $(6.49 \mu \mathrm{IU} / \mathrm{mL})$ and decreased FT4 level ( $9.19 \mathrm{pmol} / \mathrm{L})$ indicating atypical primary hypothyroidism. Moreover, as his thyroid peroxidase antibody value crossed the top detectable range $(>2000 \mathrm{IU} / \mathrm{mL})$ that confirmed Hashimoto's thyroiditis (Table 3). Several other lab factors measured were within normal range while the chest $\mathrm{X}$-ray was unremarkable. After five weeks of levothyroxine $25 \mathrm{mcg} /$ once a day, patient reported that he felt energized. Yet, his thyroid profile stood out deregulated despite it considerably progressed. ${ }^{83}$ On the other hand, this report did not provide any information on thyroid functional profiles (antibodies and TSH) performed before infection. Thus, it is also possible to speculate that Hashimoto's thyroiditis was already present before infection. Subsequently, many cases of Hashimoto's thyroiditis associated with SARS-CoV-2 infection were reported. $^{110,117,125,126}$ Besides, the duration taken from the initial respiratory-illness to Hashimoto's thyroiditis outset was shown to be very similar in other cases of autoimmune complications which were shown to be consistent with the span of "cytokine storm". ${ }^{127-131}$ Thus, the hyperinflammatory condition is often sparked by the COVID-19 disease that possibly predisposes these infected cases to get autoimmunerelated issues (Figure 3). Further, this report also reminds the physicians once again that autoimmune conditions as anticipated ailments of SARS-CoV-2, though COVID-19 cases exhibit mild symptoms.

\section{SARS-CoV-2 Propels Thyrotoxicosis}

Whether SARS-CoV-2 directly influences thyroid or COVID-19-induced "cytokine storm" triggers thyroid dysfunction is mostly unclear (Figure 3). The "cytokine 


\begin{tabular}{|c|c|c|c|c|c|}
\hline 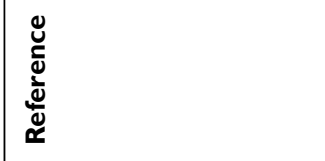 & 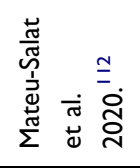 & 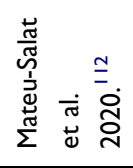 & 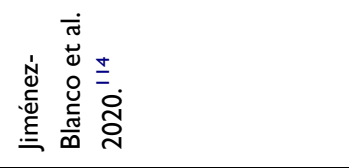 & 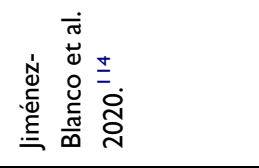 & 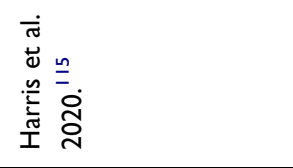 \\
\hline 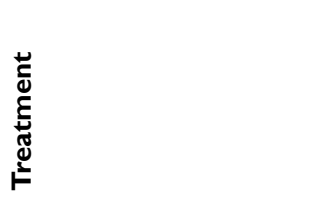 & 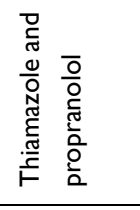 & 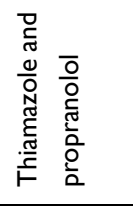 & 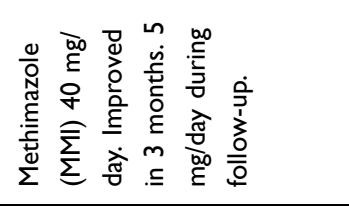 & 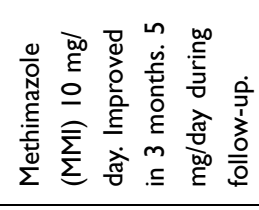 & 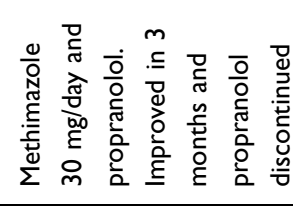 \\
\hline 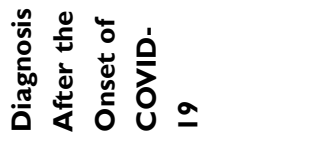 & 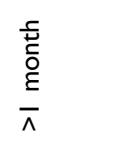 & 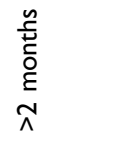 & 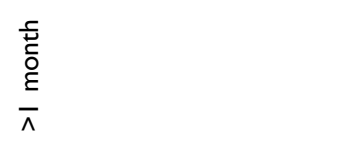 & 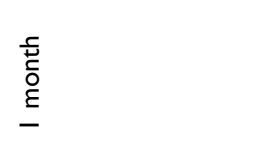 & 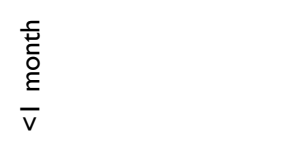 \\
\hline 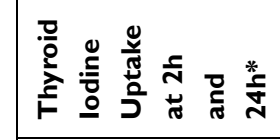 & 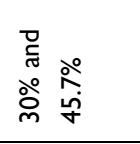 & 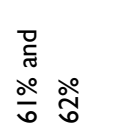 & 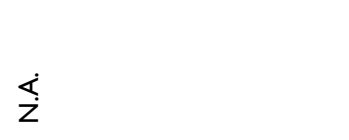 & 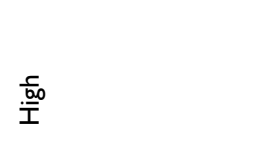 & $\begin{array}{l}\stackrel{0}{0} \\
\frac{0}{0} \\
\stackrel{0}{z}\end{array}$ \\
\hline 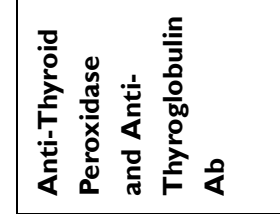 & 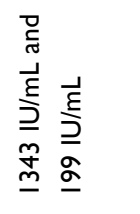 & 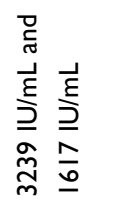 & $\dot{z}$ & ¿ & 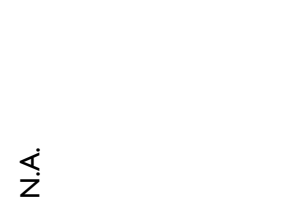 \\
\hline 咅紊 & $\frac{\vec{J}}{\frac{m}{N}}$ & 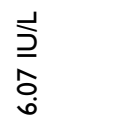 & $\hat{\infty} \underset{\sim}{\stackrel{\vec{g}}{\xi}}$ & $\frac{\underline{s}}{\underline{m}}$ & $\stackrel{\supseteq}{\supseteq}$ \\
\hline 点 & 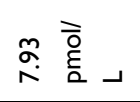 & $\dot{z}$ & ¿্் & ¿ & 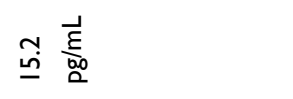 \\
\hline$\vec{r}$ & $\varrho \stackrel{\overline{\bar{o}}}{\stackrel{\bar{\varepsilon}}{a}}$ & ب & $\hat{i}=\frac{\vec{\partial}}{\hat{\lambda}}$ & $\stackrel{\circ}{\stackrel{\vec{D}}{\dot{0}}}$ & $\stackrel{\frac{\partial}{\partial}}{\stackrel{0}{0}}$ \\
\hline $\mid \frac{T}{5}$ & 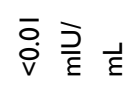 & 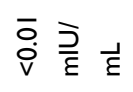 & $v \stackrel{\text { }}{\circ} \stackrel{\partial}{\circ} \bar{z}$ & v $\bar{\delta} \bar{o} \frac{\partial}{z} \vec{\varepsilon}$ & 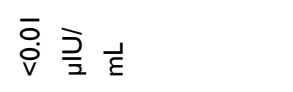 \\
\hline 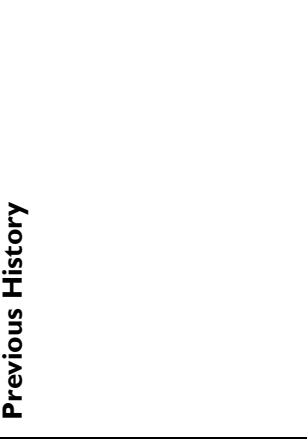 & 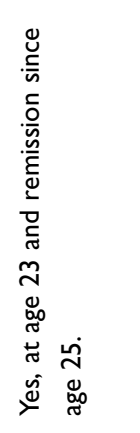 & 우 & 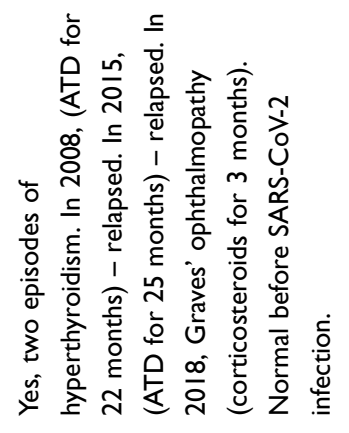 & 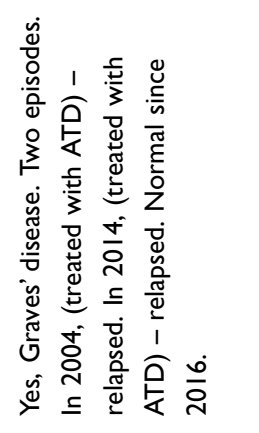 & 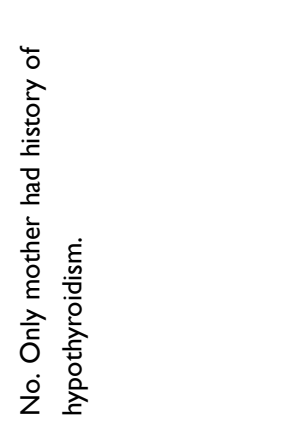 \\
\hline 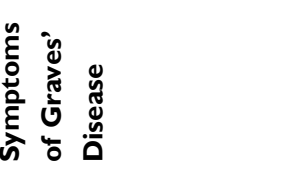 & 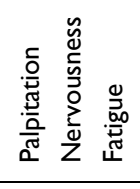 & 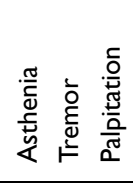 & 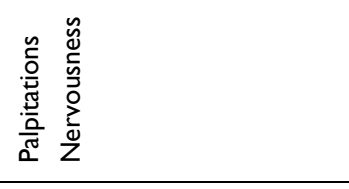 & 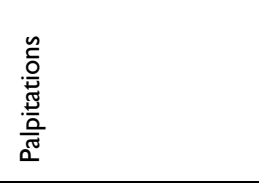 & 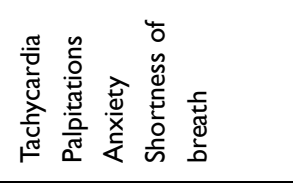 \\
\hline ڤึ & \llcorner & ᄂ & \llcorner & ч & ч \\
\hline 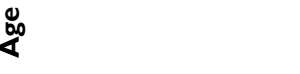 & ๑ & $\hat{n}$ & $\stackrel{\wp}{\xi}$ & $\overline{0}$ & $\bar{N}$ \\
\hline نू & - & i & $\dot{m}$ & $\dot{+}$ & เก \\
\hline
\end{tabular}




\begin{tabular}{|c|c|c|c|c|c|}
\hline 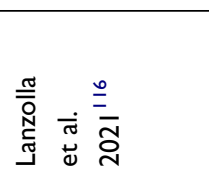 & 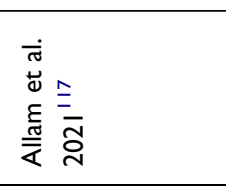 & 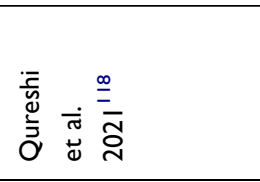 & 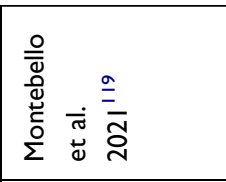 & 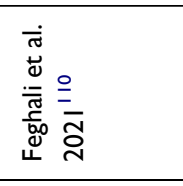 & \\
\hline 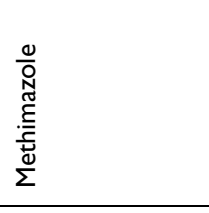 & 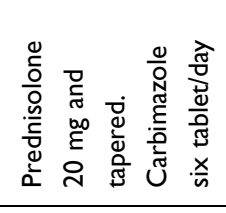 & 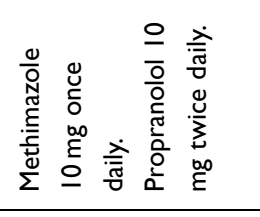 & 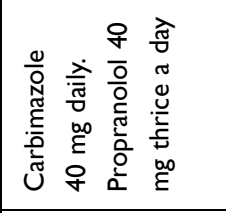 & 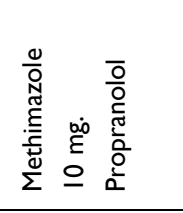 & \\
\hline 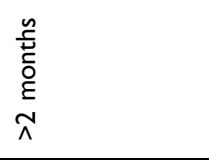 & 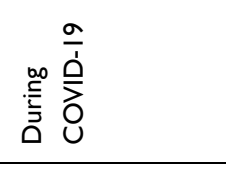 & 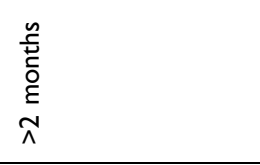 & 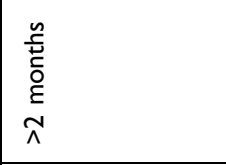 & 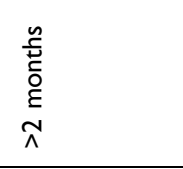 & \\
\hline $\begin{array}{l}0 \\
\frac{0}{0} \\
\frac{0}{0} \\
2\end{array}$ & 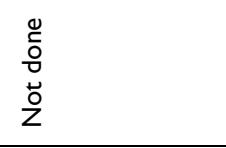 & 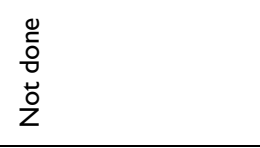 & 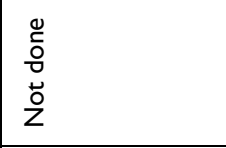 & $\stackrel{\circ}{\stackrel{\circ}{\vec{t}}}$ & 离 \\
\hline 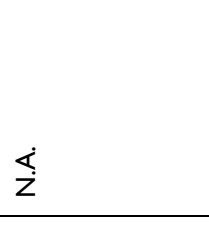 & $\frac{1}{z}$ & 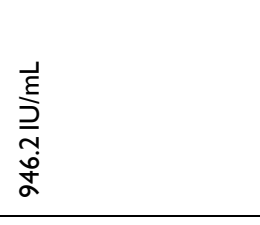 & \begin{tabular}{|l}
$\vec{E}$ \\
$\frac{\vec{E}}{D}$ \\
$\frac{O}{v}$ \\
\end{tabular} & \begin{tabular}{|l}
$\overrightarrow{\underline{\varepsilon}}$ \\
E. \\
$\infty$ \\
$\infty$ \\
$\infty$ \\
$\infty$ \\
\end{tabular} & 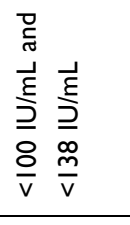 \\
\hline 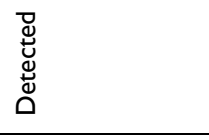 & $\underset{⿱ 乛}{د}$ & 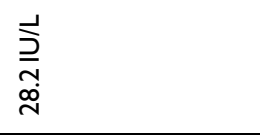 & \begin{tabular}{|l}
$\frac{1}{5}$ \\
$\frac{2}{2}$ \\
0 \\
\end{tabular} & 吕 & 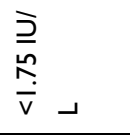 \\
\hline 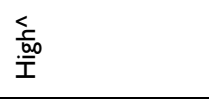 & 色 & 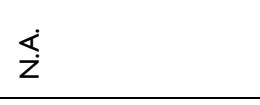 & لـ & 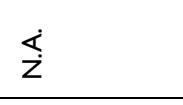 & 氙 \\
\hline 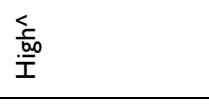 & 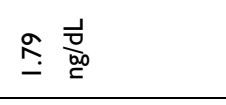 & 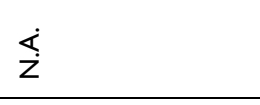 & 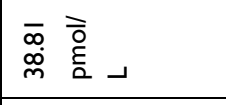 & $\overline{\mathrm{i}} \overline{\overline{\mathrm{v}}}$ & 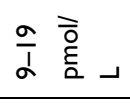 \\
\hline$\hat{\jmath}$ & $\bar{\partial} \bar{O} \frac{\partial}{\xi} \vec{\varepsilon}$ & $\overline{\grave{O}} \stackrel{\partial}{\partial} \vec{\xi}$ & $\stackrel{\circ}{\partial} \stackrel{\partial}{\partial} \vec{\Xi}$ & $\begin{array}{l}\vec{\partial} \\
\dot{0} \\
\dot{v}\end{array}$ & 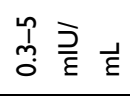 \\
\hline 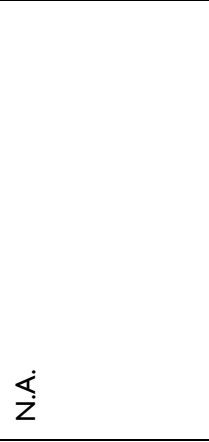 & 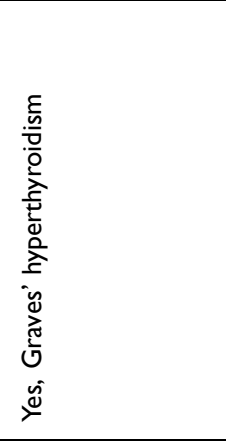 & $\stackrel{0}{z}$ & 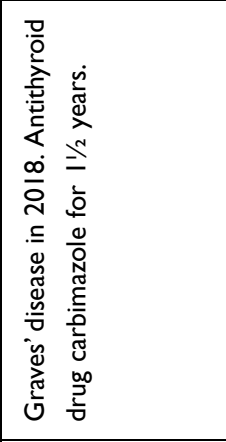 & $\stackrel{\leftrightarrow}{Z}$ & \multirow{5}{*}{ 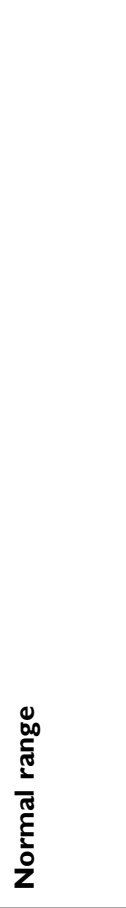 } \\
\hline 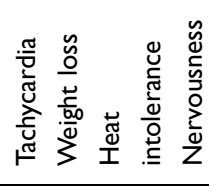 & 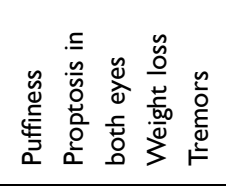 & 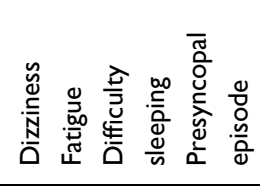 & 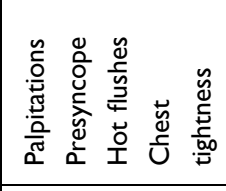 & 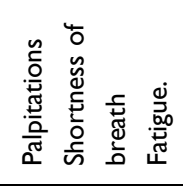 & \\
\hline 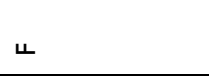 & 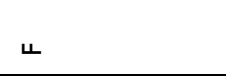 & $\Sigma$ & ч & 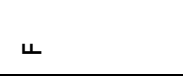 & \\
\hline$\tilde{m}$ & $\stackrel{m}{m}$ & $\underline{m}$ & z & $\tilde{m}$ & \\
\hline 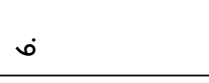 & $r$ & $\infty$ & 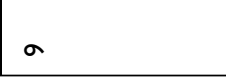 & $\stackrel{\circ}{ }$ & \\
\hline
\end{tabular}




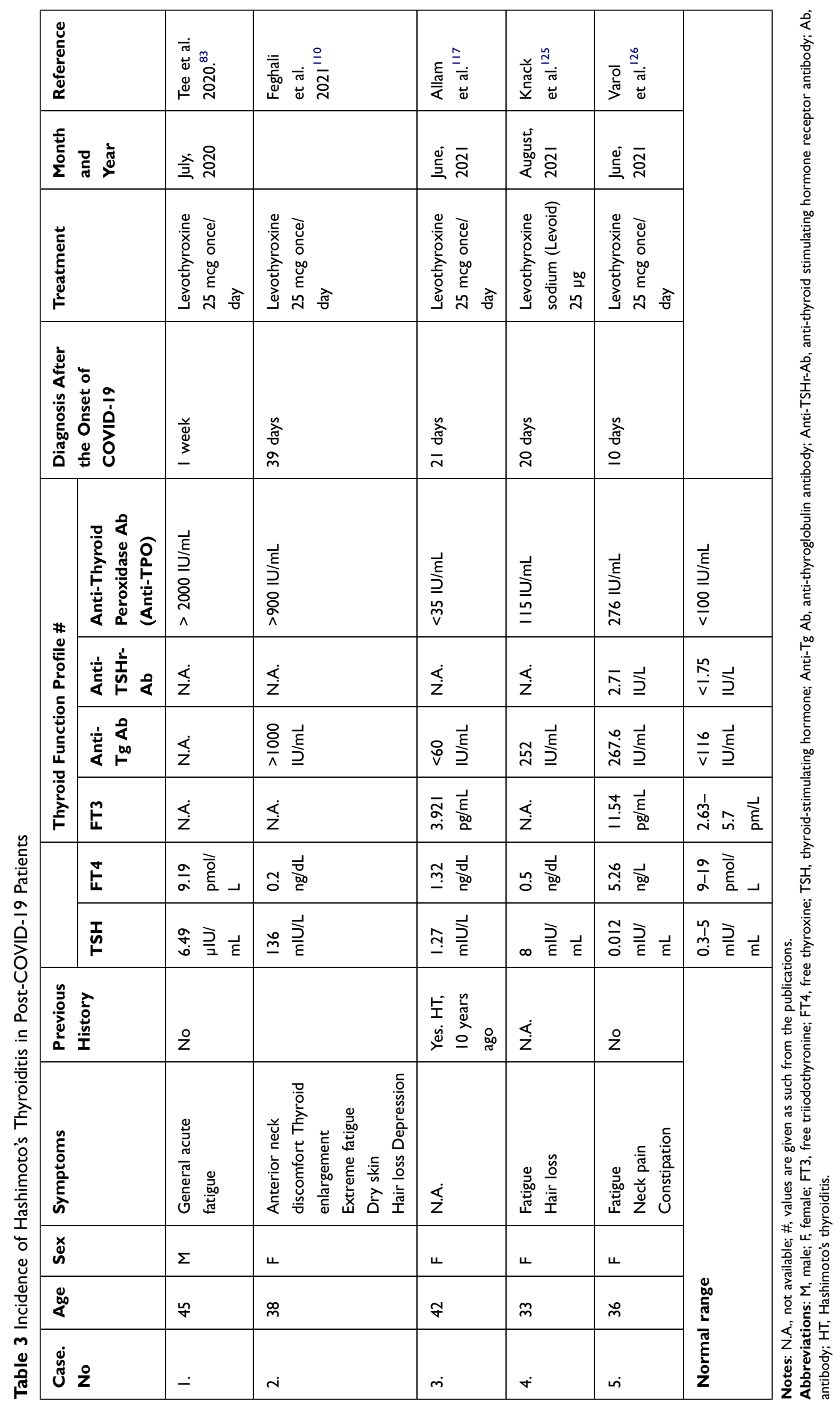


storm"-mediated effect on the thyroid has more possibilities as SARS-CoV-1 was shown to have an effect on the thyroid. Recently, a retrospective study evaluated the thyroid profiles and serum interleukin-6 (IL-6) values of 287 COVID-19 cases who were treated in non-ICU and found that 58 patients $(20.2 \%)$ were found with thyrotoxicosis (excess of thyroid hormone (TH) and low TSH), and 15 patients $(5.2 \%)$ were detected with hypothyroidism, while 214 patients $(\sim 75 \%)$ were unremarkable (normal). TSH measurements were inversely correlated to patients' age and IL-6. Besides, thyrotoxicosis was remarkably correlated to IL-6 elevation. ${ }^{82}$ These findings postulate that this may be because of SARS-CoV-2 infection-induced systemic immune activation. Another group aimed to evaluate the thyrotoxicosis prevalence in the high intensity of care unit (HICU) patients with COVID-19 positive and negative cases as it could be a chance for identifying the subacute thyroiditis cases. To rule out this, the study compared HICU patients in 2020 (HICU-20 group) and 2019 (HICU-19 group) as presence or absence of COVID19 cases, respectively. The findings showed that a considerable number of COVID-19 cases with the HICU requirement displayed thyrotoxicosis with low serum TSH apt due to SARS-CoV-2-induced subacute thyroiditis - suggestive of a non-thyroidal illness syndrome (NTIS). Likewise, HICU-20 group patients presented a similarly low number of pre-existing thyroid disorders (autoimmune and non-autoimmune) with a low prevalence when compared to the HICU-19 group. These results suggested that these thyroid issues are neither a risk factor for viral infection nor COVID-19 severances. ${ }^{76}$

\section{Triiodothyronine for Treatment of COVID-I 9 Cases}

The majority of the people recover themselves without or with minimal treatments after SARS-CoV-2 infection. Even so, a small percentage of these patients become critically ill and need hospitalization and even treatment with the support of an intensive care unit (ICU). The SARS-CoV-2 infection could induce sepsis that results in cell damage because of viral entry and its replication and hypoxia. The viral infection and hypoxia both are shown to be mediated by the p38 MAPK, a pro-apoptotic pathway. In addition, therapeutic agents such as inotropes and vasoactive drugs are often used in intensive care settings and this, in turn, activates the p38 MAPK pathway. ${ }^{21,22,81,82,132,133}$ This hyperactivated signaling enhances the mortality rate by damaging multiple vital organs regardless of current treatment options (Figure 4). Moreover, epinephrine and dobutamine are frequently used for sepsis patients howbeit were shown to be markedly interconnected with enhanced death rate and cardiac arrhythmia. ${ }^{134}$

Furthermore, a number of dysfunctions, including heart attack, shock, TH-metabolism, NTIS, etc., are frequently observed in sepsis conditions while low levels of T3 in the blood were investigated to be linked with enhanced fatality. ${ }^{135}$ Furthermore, decreased FT3 level was independently shown to predict all-cause mortality. ${ }^{77}$ In fact, the T3 was demonstrated to have cardioprotective effects in mice. ${ }^{136}$ NTIS is considered to have a physiological role in cell defense and has been identified that the thyroid hormone could enhance the endurance of cells to hypoxia events by inhibiting the p38-MAPK stimulation and accelerate tissue repairment via "controlled AKT activation". ${ }^{137}$ Thus, it was of general interest to clinically validate this effect and hence people who were subjected to angioplasty have been enrolled for a clinical trial. Furthermore, the study has already been completed but showed no adverse effects on the patients. ${ }^{137,138}$

For instance, the thyroid hormone was reported to impede herpes simplex virus infecting ability and promote host-mediated immunity machinery through enhancing natural killer (NK) cells by inducing interferon-effect on NK cells. ${ }^{139}$ Conversely, extensive use of corticosteroids in patients of ICU helps to prevent inflammation of lungs, on the other hand, reduces the immune response and clearance of the pathogen and this practice is being continued as alternatives are uncommon. This facilitated a new phase-II clinical trial to examine the consequences of high dose T3 (intravenous) in curing severely ill COVID-19 cases (Clinical Trial ID: NCT04348513).

\section{Thyroid Disease Management: Challenges During and Post-COVID- 19 Scenario}

Certainly, the COVID-19, irrespective of the first or second episode, has highly disrupted the regular stream of thyroid surgeries that enhances the patient's anxiety regarding diagnosis and prompt treatment. Moreover, the non-emergency operations and elective interventions are feasible to postpone and this raises new and unacceptable challenges. ${ }^{140}$ Particularly, mild airway compression due to large goiters could be subacute or chronic they are not symptomatic withal if that patient needs sudden intubation 


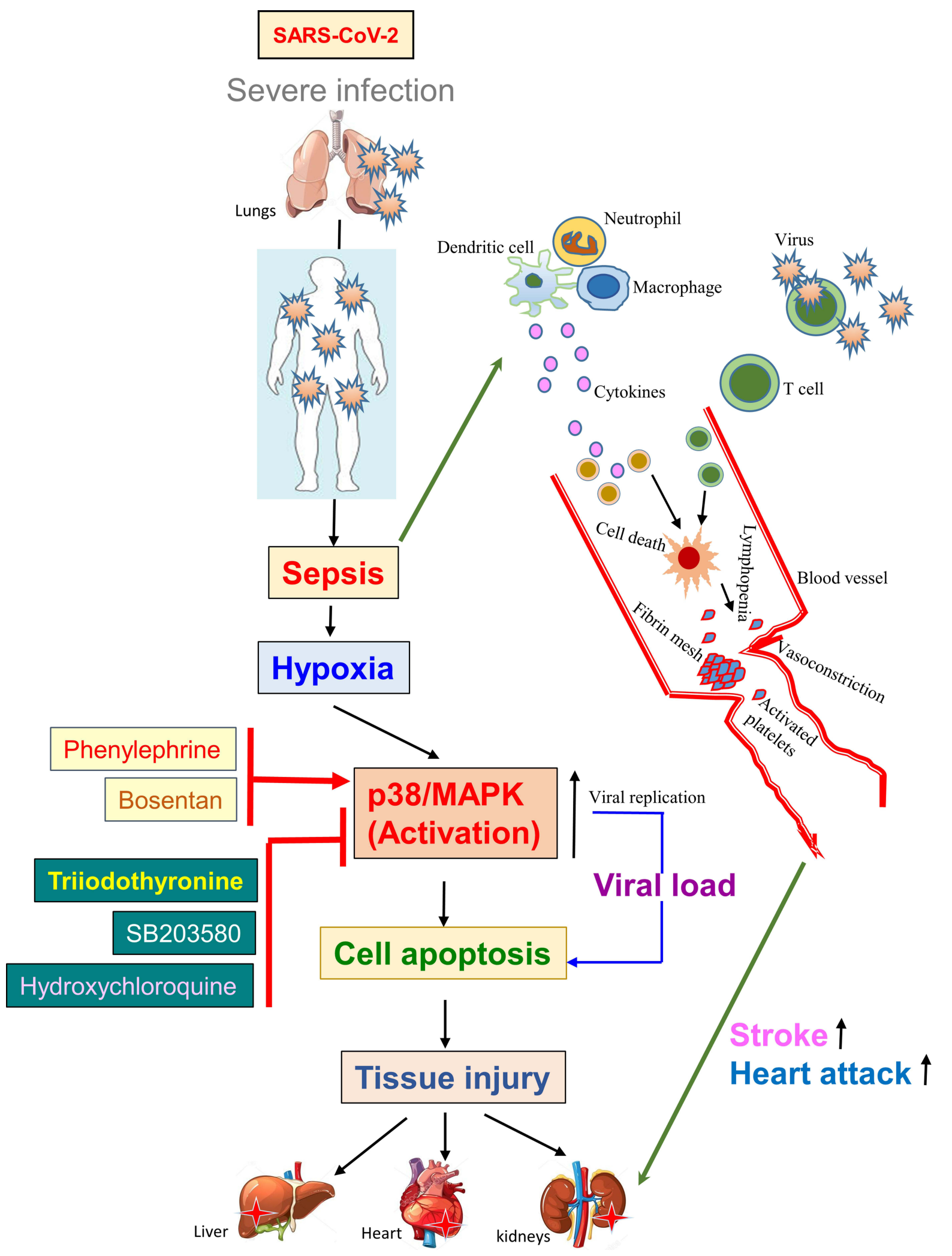

Figure 4 Triiodothyronine suppresses viral replication inhibiting the p38/MAPK pathway. The diagram shows that severe SARS-CoV-2 infection could induce sepsis, and hypoxia via activating $\mathrm{p} 38 / \mathrm{MAPK}$ resulting in "cytokine storm"-mediated cell death, platelet activation, and vasoconstriction leads to tissue injury. The triiodothyronine shows a potent antiviral effect by inhibiting the p38/MAPK pathway similar to the p38/MAPK pathway inhibitor, SB203580, and hydroxychloroquine. Triiodothyronine is currently studied in clinical trials. 
due to acute COVID-19-induced respiratory distress may be a fatal airway arrangement. ${ }^{141}$ Patients who have large goiters are more stressed and much concerned about their surgeries when differed due to the COVID-19 pandemic.

In general, autoimmune thyroiditis, surgical removal of the thyroid (total thyroidectomy), and radioisotope treatment may result in primary hypothyroidism in adults while central hypothyroidism is rarely found. The hypothyroidism patients must be supplied with enough thyroid hormones during the lockdown period and the dose of levothyroxine can be increased (30-50\%) in pregnancy cases and regularly monitored assessing the TSH and other clinical parameters to restrain the progression of hypothyroidism. ${ }^{142}$ Hypothyroidism cases infected with SARS-CoV-2 do not require any unique diagnostic and treatment options except for the continuation of the treatment of pre-existing hypothyroidism during viral infection. Recent reports showed that hypothyroidism patients did not exhibit any heightened risk of SARS-CoV-2 infection. ${ }^{143,144}$ Conversely, it is conceivable that autoimmune thyroiditis because of SARS-CoV-2 infectioninduced "cytokine storm" may develop primary hypothyroidism. On the other hand, the disrupted pituitary axis upon SARS-CoV-2 infection may cause pituitary insufficiency that may result in insufficiency in both thyroid and corticotropic which necessitates levothyroxine and hydrocortisone therapy, respectively. ${ }^{65}$

Likewise, hyperthyroidism patients with SARS-CoV-2 infection can continue ATD therapy regardless of hospitalization or home quarantine because unrestrained hyperthyroidism with COVID-19 disease may predict poor outcomes. ${ }^{124}$ Further, it is plausible that hyperthyroidism patients could be at higher risk as ACE2 - angiotensin 1-7 - Mas receptor axis was shown to be hyperactivated because of hyperthyroidism. ${ }^{113}$ Yet, future studies are warranted to ascertain this aspect.

In the post-COVID scenario, due to the long lockdown and treating primarily the COVID-19 cases in the healthcare facility, patients with other diseases including thyroid diseases are expected to highly increase. Thus, patients with likely thyroid diseases may come to the health-care facility after prolonged waiting for their slot, at least a lapse of 6-8 months with advanced thyroid disease that may lead to a worse prognosis which is also expected to enhance morbidity and mortality. Further, any deficiency in adapting these patients in the endocrine health-care facility may have a negative effect on the patient's health.

\section{Thyroid Cancer: Potential Impacts During and Post-COVID- 19 Scenario \\ Thyroid Cancer and Susceptibility to SARS-CoV-2}

Direct involvement of this virus in cancer including thyroid cancer is yet to be established. Moreover, whether thyroid cancer patients have more risk than normal people for contracting SARS-CoV-2 is unclear. Recently, it has been preliminarily observed that COVID-19 incidence was not highly frequent in thyroid cancers compared with the normal population. ${ }^{145}$ To rule out further, future studies are needed to analyze the expression of host mediators, the determinants of viral infection including ACE2, TMPRSS2, Furin in thyroid cancers when compared with normal control population while only TMPRSS2 was shown to be upregulated in human malignancies. ${ }^{146}$ However, COVID-19 poses many issues directly on viral-infected and indirectly on non-infected thyroid cancer patients.

\section{Thyroid Cancer During COVID-I 9}

Thyroid cancer patients are directly affected when they get SARS-CoV-2 infection. Whether COVID-19 disease is mild or severe in patients with thyroid cancer is not completely known. Although many articles have been published, except a few primary research reports, most of them are valuable suggestions/perspectives/viewpoints postulated towards the management of nodules and thyroid cancer during this unpredictable pandemic. ${ }^{146-151}$ Cancer patients with COVID-19 have been reported to be more fatal. ${ }^{152}$ However, in differentiated thyroid cancers (DTCs), only older age and comorbidities were statistically associated with COVID-19 severance and hospitalization than thyroid malignancy-related diagnosis, therapy, or disease burden. ${ }^{153}$ Furthermore, recent multi-center research revealed that patients with metastatic malignancies had severe COVID-19 while the non-metastatic patients displayed disease events similar to that of noncancer cases. ${ }^{154}$ This observation is yet to be replicated in progressive/metastatic thyroid malignancies.

In indirect effect, the COVID-19 poses unrivaled societal chaos, causing a hasty, open-ended shift of health-care providers worldwide. Consistently, it has been reported that the pandemic could cause significant emotional distress among thyroid cancer patients irrespective of their 
disease stage. ${ }^{155}$ Thyroid cancer diagnoses and treatments are well progressed which resulted in enhanced operative options and well-developed surveillance strategies. Nevertheless, every hospital visit of a patient for diagnosis, and operation may expose the patients and health care professionals to viruses. Without a doubt, cancer and its related management have gone virtual due to pandemic fear. A recent analysis revealed that a total of 445 outpatients with thyroid cancer were reported to be benefited from the nuclear medicine clinic over telemedicine service during the pandemic period in 2020 which was $15 \%$ less than that of the previous in-person visit of the patients to the hospital in the year, 2019 (525 cases) suggesting the requirement of a better medical telephone-network for the virtual clinic. ${ }^{156}$ The American College of Surgeons (ACS) has guided to decrease, adjourn, call off appointments, operations, invasive methods until the healthcare system can handle without fear of viral infection. On the other hand, this pandemic-related change in health-care patterns may have a chance to deny treatments to some critically ill patients that could result in an increase in morbidity and mortality. Overall, the number of thyroidectomies performed for thyroid cancers during the pandemic was reported to be relatively low compared with the previous year yet, it was not statistically significant. ${ }^{157}$ It is likely due to selective surgeries of aggressive cases than benign and uncertain malignancies. Except for the aggressive PDTCs and ATCs which progress/metastasize more rapidly, most of the DTCs and medullary thyroid cancers (MTCs) grow slowly and they are not in immediate concern. Therefore, it has been suggested that a careful evaluation of clinical profiles and assessing the rate of progression, size of the tumor, invasiveness, and proximity to the critical organ is advised in absentia in the context of COVID-19. ${ }^{158}$

Anti-cancer therapy is assumed to influence viral infection. Thus, recently numerous therapeutic strategies have been proposed for COVID-19 patients bearing thyroid cancers including DTCs, ATCs, PDTCs, MTCs in different settings include new patients, earlier diagnosed cases with indolent disease, and patients with ongoing therapy. ${ }^{146-151}$ Currently, a study reported that various anticancer treatments including immune checkpoint inhibitor, PD-1 displayed no increased risk in cancer patients with COVID19. ${ }^{159}$ Similarly, thyroid cancer therapy showed neither favorable nor unfavorable association in DTCs with concomitant COVID-19. ${ }^{153}$ Conversely, more viral infections were noticed in thyroid cancer patients with multi-kinase inhibitors (MKIs) therapy $(2.5 \%)$ compared with control cases $(0.3 \%) .{ }^{145}$ These results are inconclusive and suggest further studies in the future.

\section{Thyroid Cancer in Post-COVID-19 Scenario}

In the post-COVID scenario, because of many months of lockdown people living with asymptomatic cancer, treatment postponed micro-papillary thyroid carcinomas (micro-PTCs), and likely malignant thyroid nodules may tend to progress when treatment is compromised/postponed. This could pose more challenges such as metastatic disease, complicating the operation, and even death in some cases. This scenario is more likely to occur in the case of MTCs and some invasive/aggressive phenotypes of DTCs, ATCs, and PDTCs which is also expected to enhance morbidity and mortality. ${ }^{146-151}$ In fact, overall cases of weekly fine needle aspirations (FNAs) were reported to be decreased (62.1 to 23.1) but diagnoses of benign cases decreased while aggressive malignancies markedly raised $(6 \%)$ in the post-lockdown. ${ }^{160}$ Further, the number of thyroid cancer patients who are going to visit the health-care facility tends to be increased considerably in the post-COVID-19 scenario. Tailoring these patients based on the actual need for the immediate clinical procedure/treatment may overcome the issues and curtail the mortality rate.

\section{Conclusions and Future Prospective}

Understanding the recent advances in pathophysiological conditions in thyroid dysfunction due to SARS-CoV-2 infections clearly indicates that there is an association between viral infection and thyroid diseases and it continues to emerge as new findings rapidly come to light. The thyroid gets disrupted in both the acute phase, during the infection, and convalescence phase, post-COVID condition. Although some evidence suggests that these two conditions are either due to the direct infection of the thyroid or a "cytokine storm"-mediated autoimmune effect which in turn affects the thyroid, future studies at both molecular and clinical levels are warranted for the better understanding, diagnosis, and therapeutic management of thyroid diseases in both the acute and convalescence phases of COVID-19 patients. As high-level intensive care is required for COVID-19 patients with thyroid dysfunction, a regular thyroid function assessment can be made in COVID-19 patients at admission, during 
hospitalization, and follow-up. Moreover, the post-COVID era is the period where physicians most likely see the effect of COVID-19 on the thyroid. Physicians need to be more watchful of the possible link related to SARSCoV-2 and various thyroid diseases and other possible clinically poorly defined thyroid diseases under the causative footprint of SARS-CoV-2 infection.

\section{Acknowledgments}

The authors thank various researchers in this area whose eminent work made it a particularly enjoyable experience to write this article.

\section{Funding}

This work was not funded.

\section{Disclosure}

The authors report no conflicts of interest for this work.

\section{References}

1. Fan E, Beitler JR, Brochard L, et al. COVID-19-associated acute respiratory distress syndrome: is a different approach to management warranted? Lancet Respir Med. 2020;8(8):816-821. doi:10.1016/S2213-2600(20)30304-0

2. COVID-19 data portal, Center for Systems Science and Engineering, The Johns Hopkins University, Baltimore MD USA. Available from: https://coronavirus.jhu.edu/. Accessed October 01, 2021.

3. Li MY, Li L, Zhang Y, Wang XS. Expression of the SARS-CoV-2 cell receptor gene ACE2 in a wide variety of human tissues. Infect Dis Poverty. 2020;9(1):45. doi:10.1186/s40249-020-00662-х

4. Narayan SS, Lorenz K, Ukkat J, Hoang-Vu C, Trojanowicz B. Angiotensin converting enzymes ACE and ACE2 in thyroid cancer progression. Neoplasma. 2020;67(2):402-409. doi:10.4149/ neo_2019_190506N405

5. Wrapp D, Wang N, Corbett KS, et al. Cryo-EM structure of the 2019-nCoV spike in the prefusion conformation. Science. 2020;367(6483):1260-1263. doi:10.1126/science.abb2507

6. Kuba K, Imai Y, Rao S, et al. A crucial role of angiotensin converting enzyme 2 (ACE2) in SARS coronavirus-induced lung injury. Nat Med. 2005;11(8):875-879. doi:10.1038/nm1267

7. Tipnis SR, Hooper NM, Hyde R, Karran E, Christie, Turner AJ. A human homolog of angiotensin-converting enzyme. Cloning and functional expression as a captopril-insensitive carboxypeptidase. $J$ Biol Chem. 2000;275(43):33238-33243. doi:10.1074/jbc. M002615200

8. Donoghue M, Hsieh F, Baronas E, et al. A novel angiotensinconverting enzyme-related carboxypeptidase (ACE2) converts angiotensin I to angiotensin 1-9. Circ Res. 2000;87(5):E1-9. doi:10.1161/01.res.87.5.e1

9. Hamming I, Cooper ME, Haagmans BL, et al. The emerging role of ACE2 in physiology and disease. $J$ Pathol. 2007;212(1):1-11. doi:10.1002/path.2162

10. Vickers C, Hales P, Kaushik V, et al. Hydrolysis of biological peptides by human angiotensin-converting enzyme-related carboxypeptidase. J Biol Chem. 2002;277(17):14838-14843. doi:10.1074/jbc.M200581200
11. Walls AC, Park YJ, Tortorici MA, Wall A, McGuire AT, Veesler D. Structure, Function, and Antigenicity of the SARS-CoV-2 Spike Glycoprotein. Cell. 2020;181(2):281-292.e6. doi:10.1016/ j.cell.2020.02.058

12. Chi $\mathrm{M}$, Shi $\mathrm{X}$, Huo $\mathrm{X}, \mathrm{Wu} \mathrm{X}$, Zhang $\mathrm{P}$, Wang $\mathrm{G}$. Dexmedetomidine promotes breast cancer cell migration through Rab11-mediated secretion of exosomal TMPRSS2. Ann Transl Med. 2020;8(8):531. doi:10.21037/atm.2020.04.28

13. Glowacka I, Bertram S, Muller MA, et al. Evidence that TMPRSS2 activates the severe acute respiratory syndrome coronavirus spike protein for membrane fusion and reduces viral control by the humoral immune response. J Virol. 2011;85 (9):4122-4134. doi:10.1128/JVI.02232-10

14. Hoffmann M, Kleine-Weber H, Schroeder S, et al. SARS-CoV-2 Cell Entry Depends on ACE2 and TMPRSS2 and Is Blocked by a Clinically Proven Protease Inhibitor. Cell. 2020;181(2):271-280. e8. doi:10.1016/j.cell.2020.02.052

15. Hoffmann M, Kleine-Weber H, Pöhlmann S. A multibasic cleavage site in the spike protein of sars-cov-2 is essential for infection of human lung cells. Mol Cell. 2020;78(4):779-784.e5. doi:10.1016/j.molcel.2020.04.022

16. Daly JL, Simonetti B, Klein K, et al. Neuropilin-1 is a host factor for SARS-CoV-2 infection. Science. 2020;370(6518):861-865. doi:10.1126/science.abd3072

17. Cantuti-Castelvetri L, Ojha R, Pedro LD, et al. Neuropilin-1 facilitates SARS-CoV-2 cell entry and infectivity. Science. 2020;370(6518):856-860. doi:10.1126/science.abd2985

18. Li W, Moore MJ, Vasilieva N, et al. Angiotensin-converting enzyme 2 is a functional receptor for the SARS coronavirus. Nature. 2003;426(6965):450-454. doi:10.1038/nature02145

19. Hussain M, Jabeen N, Raza F, et al. Structural Variations in Human ACE2 may Influence its Binding with SARS-CoV-2 Spike Protein. J Med Virol. 2020;92(9):1580-1586. doi:10.1002/ jmv. 25832

20. Epelman S, Tang WHW, Chen SY, Van Lente F, Francis GS, Sen S. Detection of Soluble Angiotensin-Converting Enzyme 2 in Heart Failure: insights Into the Endogenous Counter-Regulatory Pathway of the Renin-Angiotensin-Aldosterone System. $J$ Am Coll Cardiol. 2008;52(9):750-754. doi:10.1016/j.jacc.20 08.02.088

21. Bouhaddou M, Memon D, Meyer B, et al. The Global Phosphorylation Landscape of SARS-CoV-2 Infection. Cell. 2020;182(3):685-712.e19. doi:10.1016/j.cell.2020.06.034

22. Klann K, Bojkova D, Tascher G, Ciesek S, Münch C, Cinatl J. Growth Factor Receptor Signaling Inhibition Prevents SARSCoV-2 Replication. Mol Cell. 2020;80(1):164-174.e4. doi:10.1016/j.molcel.2020.08.006

23. Bojkova D, Klann K, Koch B, et al. Proteomics of SARS-CoV-2infected host cells reveals therapy targets. Nature. 2020;583 (7816):469-472. doi:10.1038/s41586-020-2332-7

24. Facchiano A, Facchiano F, Facchiano A. An investigation into the molecular basis of cancer comorbidities in coronavirus infection. FEBS Open Bio. 2020;10(11):2363-2374. doi:10.1002/22115463.12984

25. Giovanella L, Ruggeri RM, Ovčariček PP, Campenni A, Treglia G, Deandreis D. Prevalence of thyroid dysfunction in patients with COVID-19: a systematic review. Clin Transl Imaging. 2021;1-8. doi:10.1007/s40336-021-00419-y

26. Lui DTW, Lee CH, Chow WS, et al. Thyroid Dysfunction in Relation to Immune Profile, Disease Status and Outcome in 191 Patients with COVID-19. J Clin Endocrinol Metab. 2021;106(2): e926-e935. doi:10.1210/clinem/dgaa813

27. Lui DTW, Lee CH, Chow WS, et al. Insights from a Prospective Follow-up of Thyroid Function and Autoimmunity among COVID-19 Survivors. Endocrinol Metab. 2021;36(3):582-589. doi:10.3803/EnM.2021.983 
28. Poma AM, Bonuccelli D, Giannini R, et al. COVID-19 autopsy cases: detection of virus in endocrine tissues. $J$ Endocrinol Invest. 2021:1-6. doi:10.1007/s40618-021-01628-y

29. Ferrara JL. Cytokine dysregulation as a mechanism of graft versus host disease. Curr Opin Immunol. 1993;5(5):794-799. doi:10.1016/0952-7915(93)90139-j

30. Moore JB, June CH. Cytokine release syndrome in severe COVID-19. Science. 2020;368(6490):473-474. doi:10.1126/ science.abb8925

31. Xu Z, Shi L, Wang Y, et al. Pathological findings of COVID-19 associated with acute respiratory distress syndrome. Lancet Respir Med. 2020;8(4):420-422. doi:10.1016/S2213-2600(20) 30076-X

32. Berlin DA, Gulick RM, Martinez FJ. Severe Covid-19. $N$ Engl J Med. 2020;383(25):2451-2460. doi:10.1056/NEJMcp2009575

33. Hadjadj J, Yatim N, Barnabei L, et al. Impaired type I interferon activity and inflammatory responses in severe COVID-19 patients. Science. 2020;369(6504):718-724. doi:10.1126/science. abc6027

34. Coperchini F, Chiovato L, Croce L, Magri F, Rotondi M. The cytokine storm in COVID-19: an overview of the involvement of the chemokine/chemokine-receptor system. Cytokine Growth Factor Rev. 2020;53:25-32. doi:10.1016/j.cytogfr.2020.05.003

35. Croce L, Gangemi D, Ancona G, et al. The cytokine storm and thyroid hormone changes in COVID-19. J Endocrinol Invest. 2021;44(5):891-904. doi:10.1007/s40618-021-01506-7

36. Merad M, Martin JC. Pathological inflammation in patients with COVID-19: a key role for monocytes and macrophages. Nat Rev Immunol. 2020;20(6):355-362. doi:10.1038/s41577-020-0331-4

37. Ragab D, Salah Eldin H, Taeimah M, Khattab R, Salem R. The COVID-19 Cytokine Storm; What We Know So Far. Front Immunol. 2020;11:1446. doi:10.3389/fimmu.2020.01446

38. Hue S, Beldi-Ferchiou A, Bendib I, et al. Uncontrolled Innate and Impaired Adaptive Immune Responses in Patients with COVID19 Acute Respiratory Distress Syndrome. Am J Respir Crit Care Med. 2020;202(11):1509-1519. doi:10.1164/rccm.202005$1885 \mathrm{OC}$

39. De Biasi S, Meschiari M, Gibellini L, et al. Marked T cell activation, senescence, exhaustion and skewing towards TH17 in patients with COVID-19 pneumonia. Nat Commun. 2020;11 (1):3434. doi:10.1038/s41467-020-17292-4

40. Tang Y, Liu J, Zhang D, Xu Z, Ji J, Wen C. Cytokine Storm in COVID-19: the Current Evidence and Treatment Strategies. Front Immunol. 2020;11:1708. doi:10.3389/fimmu.2020.01708

41. Ruggeri RM, Giuffrida G, Campennì A. Autoimmune endocrine diseases. Minerva Endocrinol. 2018;43(3):305-322. doi:10.237 36/S0391-1977.17.02757-2

42. Ruggeri RM, Campennì A, Deandreis D, et al. SARS-COV-2related immune-inflammatory thyroid disorders: facts and perspectives. Expert Rev Clin Immunol. 2021;17(7):737-759. doi:10.1080/1744666X.2021

43. Minciullo PL, Ruggeri RM, Vita G, Benvenga S, Gangemi S. Development of Hashimoto's thyroiditis after subacute thyroiditis: an unusual patient. Thyroid. 2009;19(1):73-74. doi:10.1089/ thy.2008.0234

44. Costela-Ruiz VJ, Illescas-Montes R, Puerta-Puerta JM, Ruiz C, Melguizo-Rodríguez L. SARS-CoV-2 infection: the role of cytokines in COVID-19 disease. Cytokine Growth Factor Rev. 2020;54:62-75. doi:10.1016/j.cytogfr.2020.06.001

45. Morshed SA, Latif R, Davies TF. Delineating the autoimmune mechanisms in Graves' disease. Immunol Res. 2012;54(1-3):191203. doi:10.1007/s12026-012-8312-8

46. Nanba T, Watanabe M, Inoue N, Iwatani Y. Increases of the Th1/ Th2 cell ratio in severe Hashimoto's disease and in the proportion of Th17 cells in intractable Graves' disease. Thyroid. 2009;19 (5):495-501. doi:10.1089/thy.2008.0423
47. Shi Y, Wang H, Su Z, et al. Differentiation imbalance of Th1/ Th17 in peripheral blood mononuclear cells might contribute to pathogenesis of Hashimoto's thyroiditis. Scand J Immunol. 2010;72(3):250-255. doi:10.1111/j.1365-3083.2010.02425.x

48. Figueroa-Vega N, Alfonso-Pérez M, Benedicto I, Sánchez-Madrid F, González-Amaro R, Marazuela M. Increased circulating proinflammatory cytokines and Th17 lymphocytes in Hashimoto's thyroiditis. J Clin Endocrinol Metab. 2010;95(2):953-962. doi:10.1210/jc.2009-1719

49. Ruggeri RM, Saitta S, Cristani M, et al. Serum interleukin-23 (IL23) is increased in Hashimoto's thyroiditis. Endocr J. 2014;61 (4):359-363. doi:10.1507/endocrj.ej13-0484

50. Ruggeri RM, Minciullo P, Saitta S, et al. Serum interleukin-22 (IL-22) is increased in the early stage of Hashimoto's thyroiditis compared to non-autoimmune thyroid disease and healthy controls. Hormones. 2014;13(3):338-344. doi:10.14310/horm.20 02.1483

51. Vitales-Noyola M, Ramos-Levi AM, Martínez-Hernández R, et al. Pathogenic Th17 and Th22 cells are increased in patients with autoimmune thyroid disorders. Endocrine. 2017;57(3):409417. doi:10.1007/s12020-017-1361-y

52. Kristensen B, Hegedüs L, Madsen HO, Smith TJ, Nielsen CH. Altered balance between self-reactive T helper (Th) 17 cells and Th10 cells and between full-length forkhead box protein 3 (FoxP3) and FoxP3 splice variants in Hashimoto's thyroiditis. Clin Exp Immunol. 2015;180(1):58-69. doi:10.1111/cei.12557

53. Li Q, Wang B, Mu K, Zhang JA. The pathogenesis of thyroid autoimmune diseases: new $\mathrm{T}$ lymphocytes - cytokines circuits beyond the Th1-Th2 paradigm. J Cell Physiol. 2019;234 (3):2204-2216. doi:10.1002/jcp. 27180

54. Afzali B, Lombardi G, Lechler RI, Lord GM. The role of T helper 17 (Th17) and regulatory T cells (Treg) in human organ transplantation and autoimmune disease. Clin Exp Immunol. 2007;148 (1):32-46. doi:10.1111/j.1365-2249.2007.03356.x

55. Giovanella L, Ruggeri RM, Petranović Ovčariček P, Campenni A, Treglia G, Deandreis D. SARS-CoV-2-related thyroid disorders: a synopsis for nuclear medicine thyroidologists. Eur J Nucl Med Mol Imaging. 2021;48(6):1719-1723. doi:10.1007/s00259-02105316-0

56. Nalbandian A, Sehgal K, Gupta A, et al. Post-acute COVID-19 syndrome. Nat Med. 2021;27(4):601-615. doi:10.1038/s41591021-01283-z

57. Carfi A, Bernabei R, Landi F; Gemelli Against COVID-19 PostAcute Care Study Group. Persistent Symptoms in Patients After Acute COVID-19. JAMA. 2020;324(6):603-605. doi:10.1001/ jama.2020.12603

58. Amenta EM, Spallone A, Rodriguez-Barradas MC, et al. Postacute COVID-19: an Overview and Approach to Classification. Open Forum Infect Dis. 2020;7(12):ofaa509. doi:10.1093/ofid/ofaa509

59. Murray T. Unpacking "long COVID”. CMAJ. 2021;193(9):E318E319. doi:10.1503/cmaj.1095923

60. Gupta A, Madhavan MV, Sehgal K, et al. Extrapulmonary manifestations of COVID-19. Nat Med. 2020;26(7):1017-1032. doi:10.1038/s41591-020-0968-3

61. Lui DTW, Lee CH, Chow WS, et al. Long COVID in Patients With Mild to Moderate Disease: do Thyroid Function and Autoimmunity Play a Role?. Endocr Pract. 2021;27(9):894902. DOI:10.1016/j.eprac.2021.06.016

62. Tsivgoulis G, Fragkou PC, Karofylakis E, et al. Hypothyroidism is associated with prolonged COVID-19-induced anosmia: a casecontrol study. J Neurol Neurosurg Psychiatry. 2021:jnnp-2021326587. doi:10.1136/jnnp-2021-326587

63. De Jongh F, Jobsis A, Elte J. Thyroid morphology in lethal nonthyroidal illness: a post-mortem study. Eur $J$ Endocrinol. 2001;144(3):221-226. doi:10.1530/eje.0.1440221 
64. Wei L, Sun S, Xu C, et al. Pathology of the thyroid in severe acute respiratory syndrome. Hum Pathol. 2007;38(1):95-102. doi:10.1016/j.humpath.2006.06.011

65. Leow MK, Kwek DS, Ng AW, Ong KC, Kaw GJ, Lee LS. Hypocortisolism in survivors of severe acute respiratory syndrome (SARS). Clin Endocrinol (Oxf). 2005;63(2):197-202. doi:10.1111/j.1365-2265.2005.02325.x

66. Chrousos GP, Kaltsas G. Post-SARS sickness syndrome manifestations and endocrinopathy: how, why, and so what? Clin Endocrinol (Oxf). 2005;63(4):363-365. doi:10.1111/j.13652265.2005.02361.x

67. Aguilera G. The Hypothalamic-Pituitary-Adrenal Axis and Neuroendocrine Responses to Stress, Handbook of Neuroendocrinology. Academic Press; 2012:175-196. doi:10.1016/B978-0-12-375097-6.10008-3

68. Wang W, Ye YX, Yao H. Evaluation and observation of serum thyroid hormone and parathyroid hormone in patients with severe acute respiratory syndrome. J Chi Antitub Asso. 2003;25:232-234.

69. Wei L, Sun S, Zhang J, et al. Endocrine cells of the adenohypophysis in severe acute respiratory syndrome (SARS). Biochem Cell Biol. 2010;88(4):723-730. doi:10.1139/O10-022

70. Fliers E, Bianco AC, Langouche L, Boelen A. Thyroid function in critically ill patients. Lancet Diabetes Endocrinol. 2015;3 (10):816-825. doi:10.1016/S2213-8587(15)00225-9

71. Van den Berghe G. Non-thyroidal illness in the ICU: a syndrome with different faces. Thyroid. 2014;24(10):1456-1465. doi:10.1089/thy.2014.0201

72. Zou R, Wu C, Zhang S, et al. Euthyroid Sick Syndrome in Patients With COVID-19. Front Endocrinol (Lausanne). 2020;11:566439. doi:10.3389/fendo.2020.566439

73. Bartalena L, Bogazzi F, Brogioni S, Grasso L, Martino E. Role of cytokines in the pathogenesis of the euthyroid sick syndrome. Eur $J$ Endocrinol. 1998;138(6):603-614. doi:10.1530/eje.0.1380603

74. Wajner SM, Maia AL. New Insights toward the Acute NonThyroidal Illness Syndrome. Front Endocrinol (Lausanne). 2012;3:8. doi:10.3389/fendo.2012.00008

75. Chen M, Zhou W, Xu W. Thyroid Function Analysis in 50 Patients with COVID-19: a Retrospective Study. Thyroid. 2021;31(1):8-11. doi:10.1089/thy.2020.0363

76. Muller I, Cannavaro D, Dazzi D, et al. SARS-CoV-2-related atypical thyroiditis. Lancet Diabetes Endocrinol. 2020;8(9):739741. doi:10.1016/S2213-8587(20)30266-7

77. Gao W, Guo W, Guo Y, et al. Thyroid hormone concentrations in severely or critically ill patients with COVID-19. J Endocrinol Invest. 2021;44(5):1031-1040. doi:10.1007/s40618-020-01460-w

78. Zhang Y, Lin F, Tu W, et al. Thyroid dysfunction may be associated with poor outcomes in patients with COVID-19. Mol Cell Endocrinol. 2021;521:111097. doi:10.1016/j.mce.2020.111097

79. Khoo B, Tan T, Clarke SA, et al. Thyroid function before, during and after COVID-19. J Clin Endocrinol Metab. 2021;106(2): e803-e811. doi:10.1210/clinem/dgaa830

80. van Gerwen M, Alsen M, Little C, et al. Outcomes of Patients With Hypothyroidism and COVID-19: a Retrospective Cohort Study. Front Endocrinol (Lausanne). 2020;11:565. doi:10.3389/ fendo.2020.00565

81. Wu D, Yang XO. TH17 responses in cytokine storm of COVID19: an emerging target of JAK2 inhibitor Fedratinib. J Microbiol Immunol Infect. 2020;53(3):368-370. doi:10.1016/j. jmii.2020.03.005

82. Lania A, Sandri MT, Cellini M, Mirani M, Lavezzi E, Mazziotti G. Thyrotoxicosis in patients with COVID-19: the THYRCOV study. Eur J Endocrinol. 2020;183(4):381-387. doi:10.1530/EJE20-0335

83. Tee LY, Hajanto S, Rosario BH. COVID-19 complicated by Hashimoto's thyroiditis. Singapore Med J. 2020. doi:10.11622/ smedj.2020106
84. Tabassom A, Edens MA. De Quervain Thyroiditis. StatPearls; 2020 .

85. Alzahrani AS, Ceresini G, Aldasouqi SA. Role of ultrasonography in the differential diagnosis of thyrotoxicosis: a noninvasive, cost-effective, and widely available but underutilized diagnostic tool. Endocr Pract. 2012;18(4):567-578. doi:10.4158/EP11170. RA

86. Ippolito S, Dentali F, Tanda ML. SARS-CoV-2: a potential trigger for subacute thyroiditis? Insights from a case report. J Endocrinol Invest. 2020;43(8):1171-1172. doi:10.1007/s40618-020-01312-7

87. Kalra P, Kumar KM, Kallur KG, Vadyanathan V, Nadig M, Shankar M. Demographic data of thyroiditis from a south Indian city. Indian J Endocrinol Metab. 2015;19(2):300-302. doi:10.4103/2230-8210.149326

88. Sweeney LB, Stewart C, Gaitonde DY. Thyroiditis: an integrated approach. Am Fam Phys. 2014;90:389-396.

89. Brancatella A, Ricci D, Viola N, Sgrò D, Santini F, Latrofa F. Subacute Thyroiditis After Sars-COV-2 Infection. $J$ Clin Endocrinol Metab. 2020;105(7):dgaa276. doi:10.1210/clinem/ dgaa276

90. Asfuroglu Kalkan E, Ates I. A case of subacute thyroiditis associated with Covid-19 infection. J Endocrinol Invest. 2020;43 (8):1173-1174. doi:10.1007/s40618-020-01316-3

91. Brancatella A, Ricci D, Cappellani D, et al. Is Subacute Thyroiditis an Underestimated Manifestation of SARS-CoV-2 Infection? Insights From a Case Series. J Clin Endocrinol Metab. 2020;105(10):dgaa537. doi:10.1210/clinem/dgaa537

92. Ruggeri RM, Campennì A, Siracusa M, Frazzetto G, Gullo D. Subacute thyroiditis in a patient infected with SARS-COV-2: an endocrine complication linked to the COVID-19 pandemic. Hormones. 2021;20(1):219-221. doi:10.1007/s42000-020-00230-w

93. Mattar SAM, Koh SJQ, Rama Chandran S, Cherng BPZ. Subacute thyroiditis associated with COVID-19. BMJ Case Rep. 2020;13(8):e237336. doi:10.1136/bcr-2020-237336

94. Guven M. Subacute Thyroiditis in the Course of Coronavirus Disease 2019: a Case Report. J Endocrinol Metab. 2020;10:110-112. doi:10.14740/jem678

95. Campos-Barrera E, Alvarez-Cisneros T, Davalos-Fuentes M. Subacute Thyroiditis Associated with COVID-19. Case Rep Endocrinol. 2020;2020:8891539. doi:10.1155/2020/8891539

96. San Juan MDJ, Florencio MQV, Joven MH. SUBACUTE THYROIDITIS IN A PATIENT WITH CORONAVIRUS DISEASE 2019. AACE Clin Case Rep. 2020;6(6):e361-e364. doi:10.4158/ACCR-2020-0524

97. Ruano R, Zorzano-Martinez M, Campos A, Rius F, Hernández M. Subacute thyroiditis might be a complication triggered by SARSCoV-2. Endocrinol Diabetes Nutr. 2020. doi:10.1016/j. endinu.2020.09.002

98. Chong WH, Shkolnik B, Saha B, Beegle S. Subacute Thyroiditis in the Setting of Coronavirus Disease 2019. Am J Med Sci. 2021;361(3):400-402. doi:10.1016/j.amjms.2020.09.011

99. Chakraborty U, Ghosh S, Chandra A, Ray AK. Subacute thyroiditis as a presenting manifestation of COVID-19: a report of an exceedingly rare clinical entity. BMJ Case Rep. 2020;13(12): e239953. doi:10.1136/bcr-2020-239953

100. Mehmood MA, Bapna M, Arshad M. A Case of Post-COVID-19 Subacute Thyroiditis. Cureus. 2020;12(12):e12301. doi:10.7759/ cureus. 12301

101. Khatri A, Charlap E, Kim A. Subacute Thyroiditis from COVID19 Infection: a Case Report and Review of Literature. Eur Thyroid J. 2021;9(6):324-328. doi:10.1159/000511872

102. Davoodi L, Oladi Z, Jafarpour H, Zakariaei Z, Soleymani E, Razavi A. A 33-year-old man with COVID-19 presented with subacute thyroiditis: a rare case report and literature review. New Microbes New Infect. 2021;41:100871. doi:10.1016/j. nmni.2021.100871 
103. Sohrabpour S, Heidari F, Karimi E, Ansari R, Tajdini A, Heidari F. Subacute Thyroiditis in COVID-19 Patients. Eur Thyroid J. 2021;9(6):321-323. doi:10.1159/000511707

104. Ghosh R, Dubey S, Sarkar A, et al. Antithyroid arthritis syndrome in a case of post-COVID-19 subacute thyroiditis. Diabetes Metab Syndr. 2021;15(3):683-686. doi:10.1016/j.dsx.2021.03.015

105. Mathews SE, Castellanos-Diaz J, Srihari A, et al. Subacute Thyroiditis and Heart Failure in a Patient Presenting With COVID-19. $J$ Investig Med High Impact Case Rep. 2021;9:23247096211009412. doi:10.1177/23247096211009412

106. Abreu R, Miguel R, Saieg M. Subacute (De Quervain) thyroiditis during the COVID-19 pandemic. Cancer Cytopathol. 2021. doi:10.1002/cncy.22449

107. Seyed Resuli A, Bezgal M. Subacute Thyroiditis in COVID-19 Patients. Ear Nose Throat J. 2021;1455613211012114. doi:10.1177/01455613211012114

108. de la Higuera López-frías M, Perdomo CM, Galofré JC. Subacute thyroiditis following COVID-19 infection. Rev Clin Esp. 2021;221(6):370-372. doi:10.1016/j.rceng.2021.01.002

109. Tjønnfjord E, Moe RB, Ghanima W, Aballi S. Subacute thyroiditis after COVID-19. Tidsskr nor Laegeforen. 2021;141(10). doi:10.4045/tidsskr.21.0169

110. Feghali K, Atallah J, Norman C. Manifestations of thyroid disease post COVID-19 illness: report of Hashimoto thyroiditis, Graves' disease, and subacute thyroiditis. $J$ Clin Transl Endocrinol Case Rep. 2021;22:100094. doi:10.1016/j. jecr.2021.100094

111. Sato D, Nishiguchi S, Tanaka E. Successful Management of Subacute Thyroiditis Following SARS-CoV-2 Infection: a Case Report. Intern Med. 2021. doi:10.2169/internalmedicine.7913-21

112. Mateu-Salat M, Urgell E, Chico A. SARS-COV-2 as a trigger for autoimmune disease: report of two cases of Graves' disease after COVID-19. $J$ Endocrinol Invest. 2020;43(10):1527-1528. doi:10.1007/s40618-020-01366-7

113. Diniz GP, Senger N, Carneiro-Ramos MS, Santos RA, BarretoChaves ML. Cardiac ACE2/angiotensin 1-7/Mas receptor axis is activated in thyroid hormone-induced cardiac hypertrophy. Ther Adv Cardiovasc Dis. 2016;10(4):192-202. doi:10.1177/ 1753944715623228

114. Jiménez-Blanco S, Pla-Peris B, Marazuela M. COVID-19: a cause of recurrent Graves' hyperthyroidism? J Endocrinol Invest. 2021;44(2):387-388. doi:10.1007/s40618-020-01440-0

115. Harris A, Al Mushref M. Graves' Thyrotoxicosis Following SARS-CoV-2 Infection. AACE Clin Case Rep. 2021;7(1):14-16. doi:10.1016/j.aace.2020.12.005

116. Lanzolla G, Marcocci C, Marinò M. Graves' disease and Graves' orbitopathy following COVID-19. J Endocrinol Invest. 2021;1-2. doi:10.1007/s40618-021-01576-7

117. Allam MM, El-Zawawy HT, Ahmed SM, Aly Abdelhamid M. Thyroid disease and covid-19 infection: case series. Clin Case Rep. 2021;9(6):e04225. doi:10.1002/ccr3.4225

118. Qureshi NK, Bansal SK. Autoimmune Thyroid Disease and Psoriasis Vulgaris after COVID-19 in a Male Teenager. Case Rep Pediatr. 2021;2021:7584729. doi:10.1155/2021/7584729

119. Montebello A. Recurrent Graves' disease post SARS-CoV-2 infection. BMJ Case Rep. 2021;14(8):e244714. doi:10.1136/bcr2021-244714

120. Prummel MF, Strieder T, Wiersinga WM. The environment and autoimmune thyroid diseases. Eur $J$ Endocrinol. 2004;150 (5):605-618. doi:10.1530/eje.0.1500605

121. Salvi M, Girasole G, Pedrazzoni M, et al. Increased serum concentrations of interleukin-6 (IL-6) and soluble IL-6 receptor in patients with Graves' disease. J Clin Endocrinol Metab. 1996;81 (8):2976-2979. doi:10.1210/jcem.81.8.8768861
122. Murugan AK, Alzahrani AS. SARS-CoV-2 plays a pivotal role in inducing hyperthyroidism of Graves' disease. Endocrine. 2021;73 (2):243-254. doi:10.1007/s12020-021-02770-6

123. Pastor S, Molina Á Sr, De Celis E. Thyrotoxic Crisis and COVID-19 Infection: an Extraordinary Case and Literature Review. Cureus. 2020;12(11):e11305. doi:10.7759/cureus.11305

124. Bartalena L, Chiovato L, Marcocci C, Vitti P, Piantanida E, Tanda ML. Management of Graves' hyperthyroidism and orbitopathy in time of COVID-19 pandemic. J Endocrinol Invest. 2020;43 (8):1149-1151. doi:10.1007/s40618-020-01293-7

125. Knack RS, Hanada T, Knack RS, Mayr K. Hashimoto's thyroiditis following SARS-CoV-2 infection. BMJ Case Rep. 2021;14(8): e244909. doi:10.1136/bcr-2021-244909

126. Varol AC, Hatice U, Gorgun S. COVID-19 and Hashimoto's Disease. Rev Assoc Med Bras. 2021;67(5):640. doi:10.1590/ 1806-9282.20210242

127. Zhang Y, Xiao M, Zhang S, et al. Coagulopathy and antiphospholipid antibodies in patients with COVID-19. $N$ Engl J Med. 2020;382(17):e38. doi:10.1056/NEJMc2007575

128. Zulfiqar AA, Lorenzo-Villalba N, Hassler $\mathrm{P}$, Andrès E. Immune thrombocytopenic purpura in a patient with COVID-19. $N$ Engl $J$ Med. 2020;382(18):e43. doi:10.1056/NEJMc2010472

129. Lazarian G, Quinquenel A, Bellal M, et al. Autoimmune haemolytic anaemia associated with COVID-19 infection. $\mathrm{Br} J$ Haematol. 2020;190(1):29-31. doi:10.1111/bjh.16794

130. Toscano G, Palmerini F, Ravaglia S, et al. Guillain-Barré Syndrome associated with SARSCoV-2. $N$ Engl $J$ Med. 2020;382(26):2574-2576. doi:10.1056/NEJMc2009191

131. Mehta P, McAuley DF, Brown M, et al. COVID-19: consider cytokine storm syndromes and immunosuppression. Lancet. 2020;395(10229):1033-1034. doi:10.1016/S0140-6736(20) 30628-0

132. Marchant D, Dou Y, Luo H, et al. Bosentan enhances viral load via endothelin-1 receptor type-A-mediated p38 mitogen-activated protein kinase activation while improving cardiac function during coxsackievirus-induced myocarditis. Circ Res. 2009;104(6):813821. doi:10.1161/CIRCRESAHA.108.191171

133. Pantos C, Mourouzis I. Translating thyroid hormone effects into clinical practice: the relevance of thyroid hormone receptor $\alpha 1$ in cardiac repair. Heart Fail Rev. 2015;20(3):273-282. doi:10.1007/ s10741-014-9465-4

134. Sato R, Ariyoshi N, Hasegawa D, et al. Effects of inotropes on the mortality in patients with septic shock. J Intensive Care Med. 2021;36(2):211-219. doi:10.1177/0885066619892218

135. Padhi R, Kabi S, Panda BN, Jagati S. Prognostic significance of nonthyroidal illness syndrome in critically ill adult patients with sepsis. Int J Crit Illn Inj Sci. 2018;8(3):165-172. doi:10.4103/ IJCIIS.IJCIIS_29_17

136. Rajagopalan V, Gorecki M, Costello C, Schultz E, Zhang Y, Gerdes AM. Cardioprotection by triiodothyronine following caloric restriction via long noncoding RNAs. Biomed Pharmacother. 2020;131:110657. doi:10.1016/j.biopha.2020.110657

137. Pantos C, Tseti I, Mourouzis I. Use of triiodothyronine to treat critically ill COVID-19 patients: a new clinical trial. Crit Care. 2020;24(1):209. doi:10.1186/s13054-020-02934-2

138. Pantos C, Kostopanagiotou G, Armaganidis A, Trikas A, Tseti I, Mourouzis I. Triiodothyronine for the treatment of critically ill patients with COVID-19 infection: a structured summary of a study protocol for a randomised controlled trial. Trials. 2020;21 (1):573. doi:10.1186/s13063-020-04474-0

139. Varedi M, Moattari A, Amirghofran Z, Karamizadeh Z, Feizi H. Effects of hypo- and hyperthyroid states on herpes simplex virus infectivity in the rat. Endocr Res. 2014;39(2):50-55. doi:10.3109/ 07435800.2013.808208 
140. Salako O, Okunade K, Allsop M, et al. Upheaval in cancer care during the COVID-19 outbreak. Ecancermedicalscience. 2020;14:ed97. doi:10.3332/ecancer.2020.ed97

141. Chen AY, Shindo M. Ethical framework for head and neck endocrine surgery in the COVID-19 pandemic. Head Neck. 2020;42 (7):1418-1419. doi:10.1002/hed.26240

142. Alzahrani AS, Al Mourad M, Hafez K, et al. Diagnosis and Management of Hypothyroidism in Gulf Cooperation Council (GCC) Countries. Adv Ther. 2020;37(7):3097-3111. doi:10.1007/s12325-020-01382-2

143. Bhatraju PK, Wurfel MM, Himmelfarb J. Trajectory of Kidney Function: the Canary in Sepsis. Am J Respir Crit Care Med. 2020;202(9):1211-1212. doi:10.1164/rccm.202007-2627ED

144. Dworakowska D, Grossman AB. Thyroid disease in the time of COVID-19. Endocrine. 2020;68(3):471-474. doi:10.1007/ s12020-020-02364-8

145. Prete A, Falcone M, Bottici V, et al. Thyroid cancer and COVID-19: experience at one single thyroid disease referral center. Endocrine. 2021;72(2):332-339. doi:10.1007/s12020-021-02650-z

146. Katopodis P, Kerslake R, Davies J, et al. COVID-19 and SARSCoV-2 host cell entry mediators: expression profiling of TMRSS4 in health and disease. Int J Mol Med. 2021;47(4):64. doi:10.3892/ ijmm.2021.4897

147. Smulever A, Abelleira E, Bueno F, Pitoia F. Thyroid cancer in the Era of COVID-19. Endocrine. 2020;70(1):1-5. doi:10.1007/ s12020-020-02439-6

148. Raghavan D, Tan AR, Story ES, et al. Management changes for patients with endocrine-related cancers in the COVID-19 pandemic. Endocr Relat Cancer. 2020;27(9):R357-R374 doi:10.1530/ERC-20-0229

149. Vrachimis A, Iakovou I, Giannoula E, Giovanella L. ENDOCRINOLOGY IN THE TIME OF COVID-19: management of thyroid nodules and cancer. Eur J Endocrinol. 2020;183 (1):G41-G48. doi:10.1530/EJE-20-0269

150. Casey RT, Valk GD, Schalin-Jäntti C, Grossman AB, Thakker RV. ENDOCRINOLOGY IN THE TIME OF COVID-19: clinical management of neuroendocrine neoplasms (NENs). Eur $J$ Endocrinol. 2020;183(2):G79-G88. doi:10.1530/EJE-20-0424

151. Scappaticcio L, Pitoia F, Esposito K, Piccardo A, Trimboli P. Impact of COVID-19 on the thyroid gland: an update. Rev Endocr Metab Disord. 2020;1-13. doi:10.1007/s11154-020-09615-Z
152. Mehta V, Goel S, Kabarriti R, et al. Case Fatality Rate of Cancer Patients with COVID-19 in a New York Hospital System. Cancer Discov. 2020;10(7):935-941. doi:10.1158/2159-8290.CD-20-0516

153. Kathuria-Prakash N, Mosaferi T, Xie M, et al. COVID-19 Outcomes of Patients With Differentiated Thyroid Cancer: a Multicenter Los Angeles Cohort Study. Endocr Pract. 2021;27 (2):90-94. doi:10.1016/j.eprac.2020.12.013

154. Dai M, Liu D, Liu M, et al. Patients with Cancer Appear More Vulnerable to SARS-CoV-2: a Multicenter Study during the COVID-19 Outbreak. Cancer Discov. 2020;10(6):783-791. doi:10.1158/2159-8290.CD-20-0422

155. Falcone R, Grani G, Ramundo V, et al. Cancer Care During COVID-19 Era: the Quality of Life of Patients With Thyroid Malignancies. Front Oncol. 2020;10:1128. doi:10.3389/ fonc. 2020.01128

156. Klain M, Nappi C, Maurea S, et al. Management of differentiated thyroid cancer through nuclear medicine facilities during Covid19 emergency: the telemedicine challenge. Eur J Nucl Med Mol Imaging. 2021;48(3):831-836. doi:10.1007/s00259-020-05041-0

157. Medas F, Ansaldo GL, Avenia N, et al.; Italian Society of Endocrine Surgery (SIUEC) Collaborative Group. Impact of the COVID-19 pandemic on surgery for thyroid cancer in Italy: nationwide retrospective study. Br J Surg. 2021;108(4):e166e167. doi:10.1093/bjs/znab012

158. Givi B, Schiff BA, Chinn SB, et al. Safety Recommendations for Evaluation and Surgery of the Head and Neck During the COVID-19 Pandemic. JAMA Otolaryngol Head Neck Surg. 2020;146(6):579-584. doi:10.1001/jamaoto.2020.0780

159. Luo J, Rizvi H, Egger JV, Preeshagul IR, Wolchok JD, Hellmann MD. Impact of PD-1 Blockade on Severity of COVID-19 in Patients with Lung Cancers. Cancer Discov. 2020;10(8):11211128. doi:10.1158/2159-8290.CD-20-0596

160. Palladino R, Migliatico I, Sgariglia R, et al. Thyroid fine-needle aspiration trends before, during, and after the lockdown: what we have learned so far from the COVID-19 pandemic. Endocrine. 2021;71(1):20-25. doi:10.1007/s12020-020-02559-z
Journal of Inflammation Research

\section{Publish your work in this journal}

The Journal of Inflammation Research is an international, peerreviewed open-access journal that welcomes laboratory and clinica findings on the molecular basis, cell biology and pharmacology of inflammation including original research, reviews, symposium reports, hypothesis formation and commentaries on: acute/chronic inflammation; mediators of inflammation; cellular processes; molecular mechanisms; pharmacology and novel anti-inflammatory drugs; clinical conditions involving inflammation. The manuscript management system is completely online and includes a very quick and fair peerreview system. Visit http://www.dovepress.com/testimonials.php to read real quotes from published authors. 